\title{
Mobilité transfrontalière et itinéraire thérapeutique des Haïtiennes et des Haïtiens de la région de Ouanaminthe
}

Cross-border mobility and therapeutic itinerary of Haitians in the Ouanaminthe region

Dominique Mathon, Philippe Apparicio et Ugo Lachapelle

\section{(2) OpenEdition}

\section{Journals}

Édition électronique

URL : http://journals.openedition.org/rfst/355

DOI : $10.4000 /$ rfst.355

ISSN : 2492-3672

Éditeur

Espaces et SOciétés (UMR 6590)

Référence électronique

Dominique Mathon, Philippe Apparicio et Ugo Lachapelle, « Mobilité transfrontalière et itinéraire

thérapeutique des Haïtiennes et des Haïtiens de la région de Ouanaminthe », Revue francophone sur la santé et les territoires [En ligne], Miscellanées, mis en ligne le 27 septembre 2019, consulté le 06 avril 2021. URL : http://journals.openedition.org/rfst/355 ; DOI : https://doi.org/10.4000/rfst.355

Ce document a été généré automatiquement le 6 avril 2021.

\section{(c) (i) (2) (2)}

La Revue francophone sur la santé et les territoires est mise à disposition selon les termes de la Licence Creative Commons Attribution - Pas d'Utilisation Commerciale - Partage dans les Mêmes Conditions 4.0 International. 


\section{Mobilité transfrontalière et itinéraire thérapeutique des Haïtiennes et des Haiitiens de la région de Ouanaminthe}

Cross-border mobility and therapeutic itinerary of Haitians in the Ouanaminthe region

Dominique Mathon, Philippe Apparicio et Ugo Lachapelle

\section{Introduction}

1 Le quotidien haïtien Le National, dans un article publié en ligne le $1^{\text {er }}$ octobre 2017, titrait «Les ressortissants haïtiens interdits de se faire soigner dans des hôpitaux publics en République dominicaine ». Selon cette publication, les importantes dépenses publiques engrangées par l'utilisation des structures de soins dominicaines par des étrangers, notamment des femmes haïtiennes enceintes, seraient à l'origine de la décision de ne plus prendre en charge gratuitement celles venues accoucher en République dominicaine. Un renforcement du contrôle frontalier de la République dominicaine aurait conduit à l'arrestation et au rapatriement, le 16 septembre 2017, de 40 ressortissants haïtiens. Ces informations relayées par la presse haïtienne font écho à celles diffusées par certains journaux dominicains qui dénoncent de manière récurrente la prise en charge par les hôpitaux publics dominicains des patient.e.s d'origine haïtienne et l'impact subséquent sur le système de santé (Acosta, 2017 ; Diario Libre, 2017 ; Le National, 2017 ; Luna, 2017 ; Luna, Santana, Rodríguez, \& Durán, 2017 ; Rivadulla, 2017 ; Saint-Pré, 2017, 2018 ; Santiago Santana, 2017).

2 Bien que la question de l'utilisation des services de santé par des Haïtiens en République dominicaine soit très largement rapportée dans certains médias dominicains, il existe peu de travaux scientifiques en Haïti et en République 
dominicaine sur le recours transfrontalier aux soins de santé. Plusieurs auteurs soulignent néanmoins l'existence d'une mobilité transfrontalière plus ou moins intense dans le domaine de la santé (Dilla Alfonso, 2004, 2007, 2011 ; Dilla Alfonso et al., 2010 ; Guerrero, Donastorg, \& de los Santos, 2014). Cependant, aucune mesure de celle-ci ne semble être disponible à l'exception des statistiques peu détaillées produites par le Ministère de la Santé publique (MSP) sur l'utilisation des services de santé dominicains par les étrangers. De plus, les motifs d'une telle mobilité sont rarement analysés. Pour certains, la carence de services en Haïti serait à l'origine de ce recours (Dilla Alfonso, 2011 ; Guerrero et al., 2014 ; Poschet El Moudden, 2006) ainsi que la gratuité des soins en République dominicaine (Guerrero et al., 2014). Cette mobilité s'inscrit par ailleurs dans la dynamique des relations entre Ouanaminthe (Haïti) et Dajabón (République dominicaine) (Dilla Alfonso, 2004). Pourtant, traverser la frontière, même aux points de passage officiels, peut s'avérer compliqué pour les Haïtien.ne.s. En effet, plusieurs auteurs soulignent des pratiques abusives et agressions (physiques ou verbales) dont sont victimes les Haïtiennes (le plus souvent) et les Haïtiens à la frontière (Murray, 2010a ; Petrozziello \& Wooding, 2014) ou au marché dit «binational» de Dajabón. Comment alors expliquer, dans un environnement empreint de tensions et d'agressivité, cette mobilité transfrontalière en santé ? Quels sont les déterminants du recours transfrontalier aux soins? À quelle étape de son itinéraire thérapeutique, le patient décide de se faire soigner en République dominicaine? Quel est le profil des personnes qui se rendent en République dominicaine pour des soins?

Cette recherche se situe dans la lignée des travaux effectués sur la mobilité transfrontalière en santé, notamment entre pays à faible revenu ou entre pays émergents (Bochaton, 2015 ; Dione, 2013 ; Kangas, 2007 ; Ormond \& Sulianti, 2017). Elle explore à partir d'entretiens l'itinéraire thérapeutique de patient.e.s d'origine haïtienne $(\mathrm{N}=21)$ se rendant à Dajabón (République dominicaine) pour des soins. La recherche vise à cerner les motifs du recours aux soins transfrontaliers, ainsi que les facteurs et acteurs influençant la prise de décision. À la suite d'une revue de littérature sur la mobilité transfrontalière, un bref portrait de la frontière haïtiano-dominicaine avec un accent particulier sur le doublet Ouanaminthe-Dajabón est dressé. Puis, la méthodologie et les principaux résultats sont présentés et discutés.

\section{De la notion de mobilité transfrontalière et ses déterminants}

4 La littérature sur la mobilité internationale des patient.e.s est plutôt dense; elle témoigne de l'ampleur et de la diversité du phénomène à l'échelle mondiale. Ces déplacements à des fins thérapeutiques soulignent, à certains égards, les disparités entre pays dans l'offre de soins; des disparités que la mondialisation de la santé, bien qu'ayant contribué à une certaine uniformisation des pratiques et des traitements, n'a pas su enrayer (Bochaton, 2009; Fassin, 2001). Elles s'inscrivent dans le processus en cours de la marchandisation des soins de santé tout en mettant l'accent sur des pratiques individuelles transnationales qui tendent à une certaine reconfiguration des systèmes de santé. En d'autres termes, la mobilité des patients et patientes remet en question le principe de territorialité sur lequel est construit le système de santé (Durham, 2017 ; Glinos, Baeten, Helble, \& Maarse, 2010). 
5 Le recours aux soins de santé au-delà des frontières nationales concerne autant les pays à haut revenu que ceux à moyen et faible revenu, avec des particularités selon le contexte, les situations migratoires, les caractéristiques socioéconomiques et culturelles (Laugesen \& Vargas-Bustamante, 2010). Les résultats des différentes études portant sur la question soulignent la diversité des flux : 1) «tourisme médical», appellation controversée caractérisant notamment les flux de pays à revenu élevé vers ceux à plus faible revenu (Bochaton \& Lefebvre, 2008; Connell, 2015);2) « retours médicaux" (medical returns en anglais) des immigrants vers leur pays d'origine (Bergmark, Barr, \& Garcia, 2010 ; De Jesus \& Xiao, 2013 ; Glinos, Doering, \& Maarse, 2012 ; Horton \& Cole, 2011; Lee, Kearns, \& Friesen, 2010) ; 3) voyages médicaux de patients et patientes de pays à faible revenu vers des pays émergents ou à faible ou moyen revenus (Kangas, 2007) et/ou disposant d'une offre médicale attrayante (technologie développée, services spécialisés, etc.); 4) mobilité transfrontalière caractéristique des dynamiques entre pays se partageant une frontière (Allen, 2013 ; Bochaton, 2009; Byrd \& Law, 2009; Caggiano, 2007 ; Dione, 2013; Durham, 2017; Liberona Concha, Tapia Ladino, \& Contreras Gatica, 2017).

6 Les motifs à l'origine de ces déplacements sont multiples. Des variations peuvent être notées selon la zone d'étude, la direction des flux ou les caractéristiques socioéconomiques des personnes concernées. Néanmoins, il ressort que la disponibilité, l'accessibilité économique ou financière, la familiarité et la perception de la qualité des services influencent la prise de décision (Glinos et al., 2010). L'attractivité des soins et la proximité peuvent, en contexte frontalier, générer une polarisation des flux (Bochaton, 2009; Dione, 2013). Plusieurs études soulignent par ailleurs le poids des réseaux sociaux, de facteurs ethnolinguistiques dans la décision de voyager (Bochaton, 2009; Durham, 2017 ; González-Vázquez, Torres-Robles, \& Pelcastre-Villafuerte, 2013) ou de retourner à son pays d'origine (Glinos et al., 2012) pour des soins. L'insatisfaction par rapport à l'expérience des soins reçus localement et l'espoir d'une meilleure prise en charge interviennent également (Glinos et al., 2010; Horton \& Cole, 2011; Liberona Concha et al., 2017; Wismar, Palm, Figueras, Ernst, \& Van Ginneken, 2011). Ceci entraîne dans certains cas des itinéraires thérapeutiques cumulant le recours aux soins à l'intérieur et à l'extérieur de leur pays (Lee et al., 2010; Sakoyan, 2012). Ces déplacements volontaires de courte durée, parfois le temps d'une consultation notamment dans le cas de la mobilité transfrontalière, s'inscrivent dans le processus mis en place par l'individu et/ou sa famille pour résoudre un problème de santé.

\section{Itinéraire thérapeutique et mobilité transfrontalière}

7 L'itinéraire thérapeutique (health seeking process), soit le cheminement des personnes dans leur quête de guérison surtout quand la maladie est chronique ou récurrente, est sinueux et complexe (Benoist, 1993 ; Chrisman, 1977 ; Janzen \& Arkinstall, 1995). Il l'est d'autant plus, en contexte de pluralisme médical (Benoist, 1993 ; Fainzang, 1985 ; Fosso, 2011 ; Janzen \& Arkinstall, 1995) ou d'offre de «thérapies alternatives » (Marcellini, Turpin, Rolland, \& Ruffié, 2000) où coexistent biomédecine, médecines alternatives et médecines traditionnelles. Dès lors, l'itinéraire thérapeutique peut mobiliser les différents recours disponibles (biomédecine, thérapies ou médecines alternatives, automédication, etc.) (Benoist, 1993; Janzen \& Arkinstall, 1995; Marcellini et al., $2000 ; \mathrm{J}$. Tremblay, 1995). C'est un «mouvement circulaire et continu où le patient 
articule différents contenus en une «médicalité unique» » (Fosso, 2011, p. 2). Plusieurs éléments interviennent et interagissent tout au long de l'itinéraire thérapeutique comme les expériences passées, les interactions avec des tiers/pairs, les représentations de la maladie, l'offre de soins, les caractéristiques socioéconomiques individuelles (Chrisman, 1977 ; Fainzang, 1985 ; Fassin, 1990).

Quelques études s'intéressant à la mobilité transfrontalière ou transnationale en santé abordent de manière spécifique l'itinéraire thérapeutique des patients en soulignant l'importance du capital social (Bochaton, 2009, 2015 ; Durham, 2017 ; Kangas, 2007). Les réseaux sociaux influencent et interviennent tout au long de l'itinéraire thérapeutique (Bochaton, 2009, 2015). De manière spécifique, ils orientent et guident les choix en permettant le partage des informations diverses sur les établissements de santé, leur fonctionnement, etc. Ils peuvent aussi appuyer financièrement ou moralement, le cas échéant. Bochaton $(2009,2015)$ montre aussi l'importance du capital spatial, soit de l'expérience et de la connaissance de la frontière, dans ces déplacements thérapeutiques transfrontaliers. Quant à Durham (2017), il estime que la mobilité thérapeutique transfrontalière résulte de la capacité de mobilisation et de l'interaction des réseaux sociaux et économiques et du capital culturel des individus.

Focaliser sur l'itinéraire thérapeutique permet d'explorer les logiques et comprendre le «butinage médical» (Fosso, 2011) des patient.e.s, soit le cumul de différentes offres thérapeutiques. La notion de "butinage " est empruntée à un travail d'anthropologie sociale portant sur les pratiques religieuses plurielles au Brésil (Droz, Oro, \& Soares, 2014 ; Droz, Soares, Gez, \& Rey, 2016 ; Soares, 2009, 2011). Elle permet d'interroger le comportement des individus afin d'en cerner le sens et les logiques sociales qui les sous-tendent. Ceci en tenant compte que le contexte socioéconomique et politique, les particularités de la frontière, le fonctionnement des différents systèmes de santé influencent aussi le comportement des individus. Il faut aussi considérer que les inégalités observées dans l'accès aux soins révèlent autant des problèmes d'accessibilité physique, financière que ceux liés à l'existence d'une offre de soins de qualité (Fassin, 1990).

\section{La frontière haïtiano-dominicaine : entre asymétrie et interdépendance}

\section{Une dynamique frontalière complexe tissée à l'aune d'un passé et d'un présent empreints de conflits et de solidarités}

Haïti et la République dominicaine sont parmi les rares pays insulaires qui partagent une frontière internationale. L'élaboration et l'inscription de la frontière haïtianodominicaine dans l'espace est autant liée à l'histoire coloniale de l'île qu'au processus de formation et de consolidation des deux États-nations (Corten, 2011 ; Fouchard, 2017 ; Hector \& Hurbon, 2009a ; Moya Pons, 2002, 2009 ; Price-Mars, 1953; Théodat, 2003). En Haïti, la révolte des esclaves conduit à l'indépendance (1804) et mène à la création d'un État anticolonial, anti-esclavagiste et antiraciste ainsi qu'au rejet du système de plantation (Hector \& Hurbon, 2009b). La République dominicaine prend son indépendance d'Haïti (1844). De ces trajectoires différentes, résulte une «double insularité » (Théodat, 2003) qui se manifeste dans le système sociospatial, dans les références culturelles, dans les structures économiques et politiques. 
11 La frontière a été tout au long de l'histoire de ces deux pays (et ce depuis l'époque coloniale) autant une zone de contact qu'une barrière ou une zone de convoitise. Ouanaminthe (Haïti) et Dajabón (République dominicaine) émergent d'ailleurs à la faveur des pratiques commerciales entre les populations des deux rives de la rivière Massacre et ce, par-delà les rivalités entre les empires coloniaux français et espagnols. Leur histoire est celle de pratiques de bon voisinage et de coexistence harmonieuse mais aussi celle d'un "contentieux historique », le massacre en 1937 en République dominicaine de dizaines de milliers d'Haïtiens (plus de trois mille victimes dans la ville de Ouanaminthe selon Jan (1950)). Après ce massacre, des tensions naissent dans les relations entre les deux pays avec pour corollaire une diminution dans l'intensité des échanges transfrontaliers, une séparation des familles haïtiano-dominicaines. Aujourd'hui, le passage Dajabón-Ouanaminthe, l'un des quatre points d'entrée officiels (figure 1) est le deuxième en importance au regard de l'intensité des flux (personnes et biens). Ce poste frontalier est d'ailleurs considéré comme une plaque tournante des échanges transfrontaliers (Théodat, Mathon, Mathelier, \& Casséus, 2003).

Figure 1 : La frontière haïtiano-dominicaine

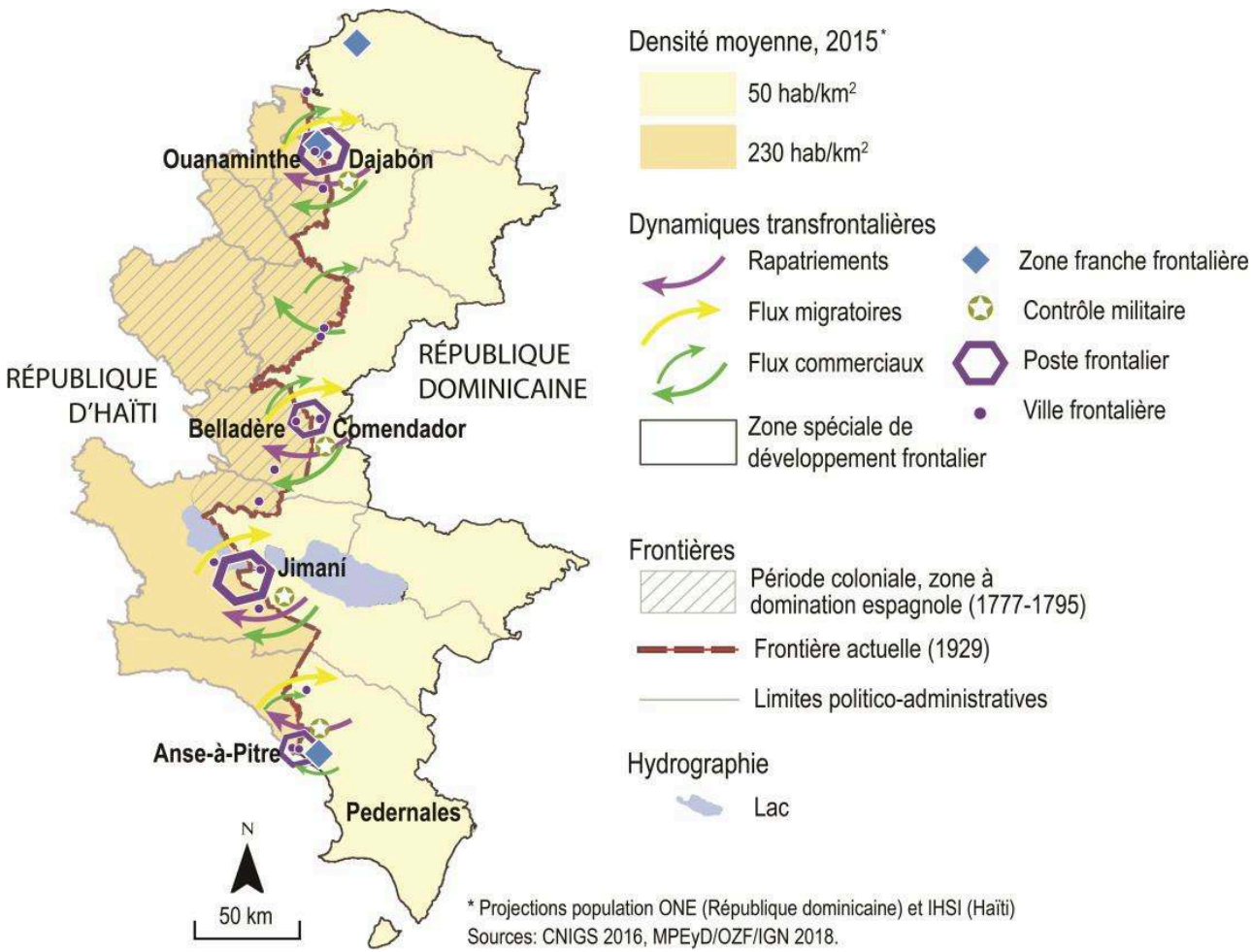

Les données disponibles sur les flux de personnes ne renseignent que sur les contrôles migratoires (entrées et sorties) (figure 2) et non sur les navettes quotidiennes (travail, école, approvisionnement, etc.) ou les jours de marché. Le marché «binational » de Dajabón accueille deux fois par semaine, les lundi et vendredi, un nombre imposant d'acheteurs haïtiens et dans une moindre mesure de vendeurs à cause des contrôles et restrictions de toute sorte (réglementations, exactions, confiscations de marchandises, etc.) à l'encontre de ces derniers. Le contrôle douanier du côté haïtien vis-à-vis des marchandises dominicaines est beaucoup moins strict. Il est à noter que pour les Haïtien.ne.s, l'obtention d'un visa est nécessaire pour traverser la frontière, à l'exception des jours de marché où la traversée peut se faire librement pour se rendre 
au marché. Les frontaliers - les personnes vivant dans la zone frontalière haïtienne peuvent, s'ils le désirent, faire la demande d'un carnet (valide un an) qui les autorise à traverser et mener des activités économiques (petits commerces) dans les limites du marché « binational».

Figure 2 : Flux migratoires entre les deux pays selon les quatre postes frontaliers

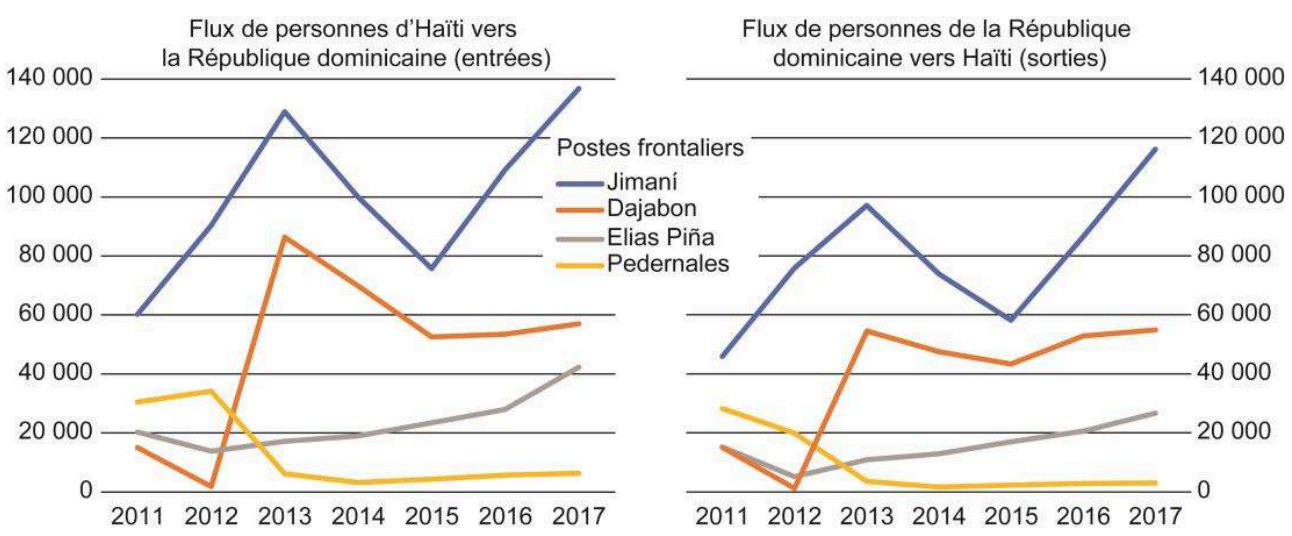

Source : Direction générale de la migration de la République dominicaine.

13 Les apports de main-d'œuvre haïtienne à l'économie dominicaine datent du début du $20^{e}$ siècle, à partir de l'occupation américaine des deux pays. Les immigrants haïtiens représentent, en 2017, 87\% de la population immigrante en République dominicaine (Oficina Nacional de Estadistica, 2018). Cette forte migration pose par ailleurs la question de l'utilisation des services de santé publique. La proportion d'Haïtiens et Haïtiennes reçus en consultation en 2017 représente 83\% des patients étrangers. Cependant, ces consultations, rapportées à l'ensemble de celles réalisées par les établissements de santé publique dominicains, ne comptent que pour environ $5 \%$ des consultations réalisées (Servicio Nacional de Salud, 2017).

Les statistiques de l'hôpital public Ramón Matías Mella situé à Dajabón renseignent cependant un peu mieux sur l'utilisation de ses services par des patient.e.s d'origine haïtienne. Ainsi, selon les données disponibles pour 2014-2016, les Haïtien.ne.s auraient fréquenté cette structure de soins pour des maladies comme la tuberculose, la malaria, le choléra, la dengue (figure 3). De plus, 35\% des femmes ayant accouché à l'hôpital Ramón Matías Mella sont d'origine haïtienne (figure 3). 
Figure 3 : Cas traités à l'hôpital provincial de Dajabón de 2014 à 2016

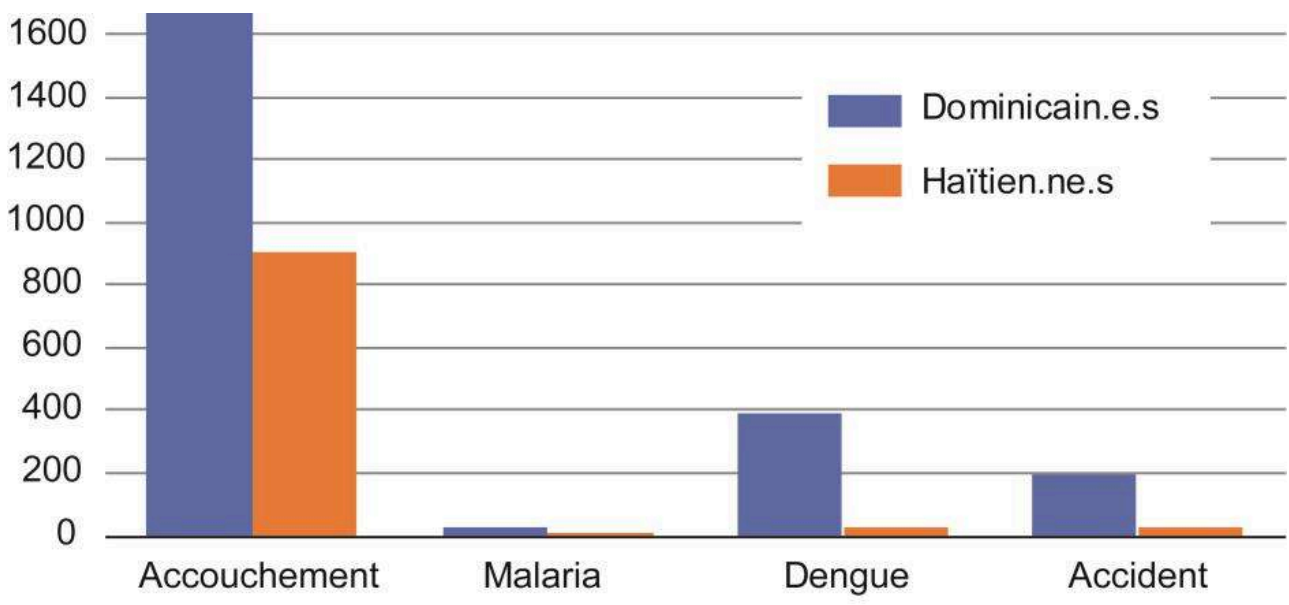

Source: Hôpital Ramón Matías Mella de Dajabón

15 Sur l'ensemble des patient.e.s haïtiennes venues accoucher, la moitié d'entre elles résident en Haïti et l'autre moitié en République dominicaine. Il convient de relever le fait que: i) plus des deux tiers $(68 \%)$ des patient.e.s sont originaires de localités frontalières situées au sud de Ouanaminthe ; ii) celles-ci ont été référées à l'hôpital de Dajabón par des centres de santé dominicains - proche de leur lieu de résidence - où elles s'étaient rendues initialement. Parallèlement, $32 \%$ d'entre elles viennent de différentes villes haïtiennes (figure 4) : Ouanaminthe, Fort-Liberté, Trou du Nord, CapHaïtien, Gonaïves, et même Port-au-Prince (la capitale) située à plus de $300 \mathrm{~km}$. Dans ce cas les patient.e.s se rendent directement à l'hôpital de Dajabón pour leur accouchement. Cela dit, des informations complémentaires auraient été nécessaires pour mieux cerner les raisons portant des femmes à faire un aussi long trajet notamment dans les cas de résidence déclarée à Port-au-Prince ou au Gonaïves $(162 \mathrm{~km})$. 
Figure 4 : Distribution selon le lieu de résidence déclaré des patientes haïtiennes ayant accouché à l'hôpital de Dajabón entre 2014 et 2016
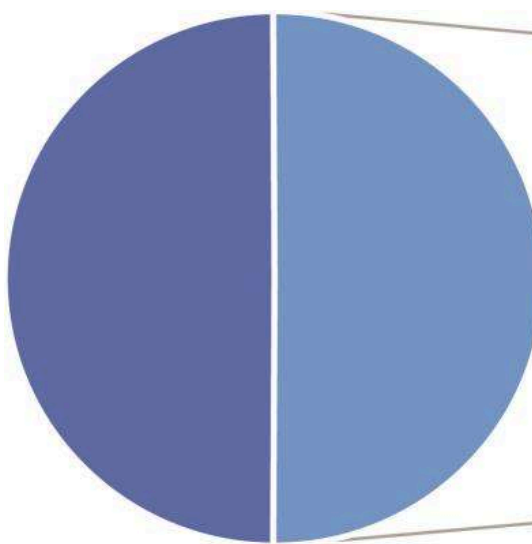

République dominicaine

République d'Haïti

Source: Hôpital Ramón Matías Mella de Dajabón

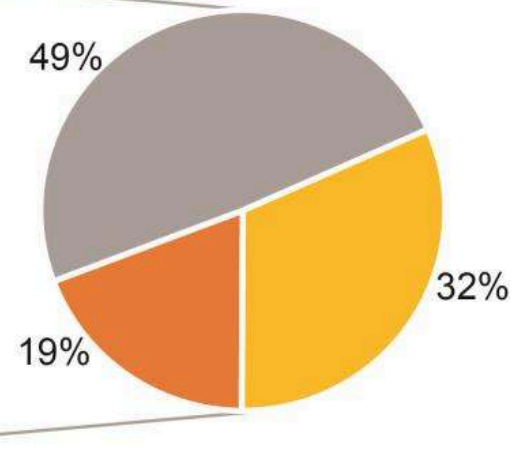

Tilori

Capotille

Plusieurs villes haïtiennes dont Ouanaminthe pour le moins contrastée allant du rejet à l'acceptation: discours l'assimilant à une «invasion », acceptation utilitaire (bénéfice économique), stéréotypes empreints de préjugés antihaïtiens, compassion (Dilla Alfonso, 2011; Murray, 2010a). Dilla Alfonso (2011) souligne que de toutes les villes frontalières, Dajabón est celle où la perception à l'encontre des Haïtiens est la plus "ambiguë et inconsistante». Il existe aussi une perception négative des Dominicains notamment chez les Haïtiens et Haïtiennes des villes frontalières haïtiennes; celle-ci est fondée sur leur expérience de la frontière (abus, agressions verbales et physiques, exactions, confiscation de marchandises, etc.) (Murray, 2010a, 2010b).

17 Les contradictions et l'asymétrie des interactions soulignent les enjeux de la redéfinition de l'espace frontalier pour en faire un "espace de coexistence et de coopération » (Dilla Alfonso et al., 2010 ; Petrozziello \& Wooding, 2014 ; Wooding, 2012).

\section{Des écarts dans l'offre de soins et les ressources disponibles}

Au cours des années 1990, une réforme du système de santé a été initiée en Haïti en vue d'améliorer l'accès à la santé et le rendre plus équitable. Une structure pyramidale composée de trois niveaux de soins (primaire, secondaire et tertiaire) avec un système de référence et contre-référence a été mise en place (figure 5). L'offre de soins est assurée par les secteurs public et privé. 
Figure 5 : Pyramide de soins

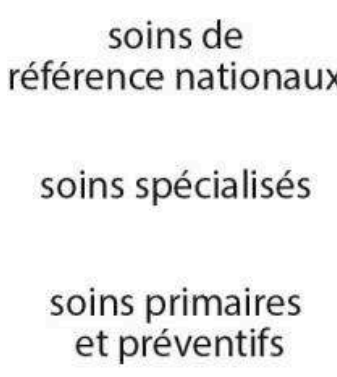

$\begin{array}{ll}\text { HS } & \text { Hôpital spécialisé et universitaire } \\ \text { HD } & \text { Hôpital départemental } \\ \text { HCR } & \text { Hôpital communautaire de référence } \\ \text { CS } & \text { Centre de santé } \\ \text { CCS } & \text { Centre communataire de santé }\end{array}$

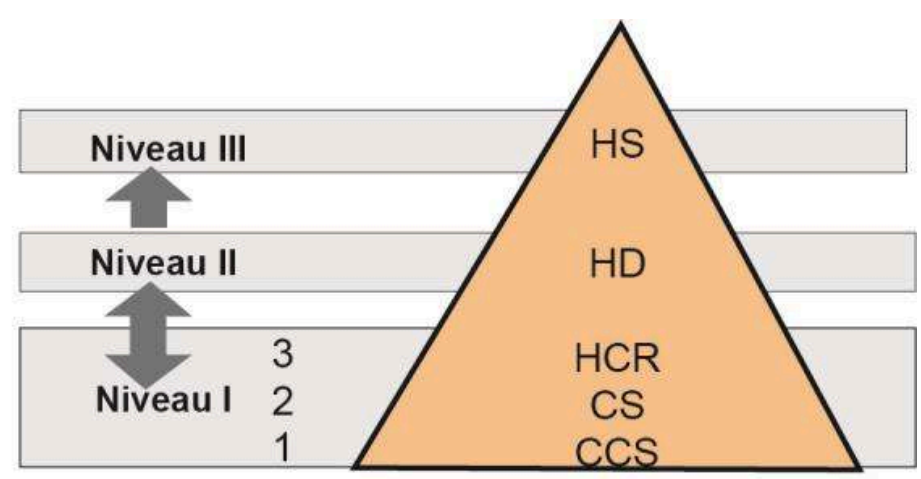

Les statistiques récentes (2017) du Ministère de la Santé publique et de la population (MSPP) en Haïti révèlent une forte présence du secteur privé à but lucratif dans le secteur de la santé. En effet, environ 30\% des établissements au niveau national sont administrés par le secteur privé à but lucratif, $17 \%$ par le secteur privé à but non lucratif et $19 \%$ comptent un système de gestion mixte (privé et public) (Ministère de la Santé Publique et de la Population, 2018). La situation en matière d'offre de soins à Ouanaminthe ne diffère pas de celle observée sur l'ensemble du territoire national. Selon les données collectées sur le terrain (2016-2017) et celles du MSPP, le secteur privé domine largement. En effet, sur les sept structures de soins identifiées (figure 6) : six sont privées et une publique. Le centre de santé de Ouanaminthe qui fait office, selon des responsables du MSPP, d'hôpital communautaire de référence (HCR) ne compte pas pour autant tous les services correspondant à cet échelon (notamment ceux relatifs à la médecine interne). Le Centre de santé de Ouanaminthe offre les services de base : médecine générale, pédiatrie, gynécologie, VIH et Tuberculose, centre de choléra, réhabilitation, maternité, imagerie (échographie et rayon $\mathrm{X}$ ), salle d'opération pour gynécologie/obstétrique, urologie, laboratoire. 


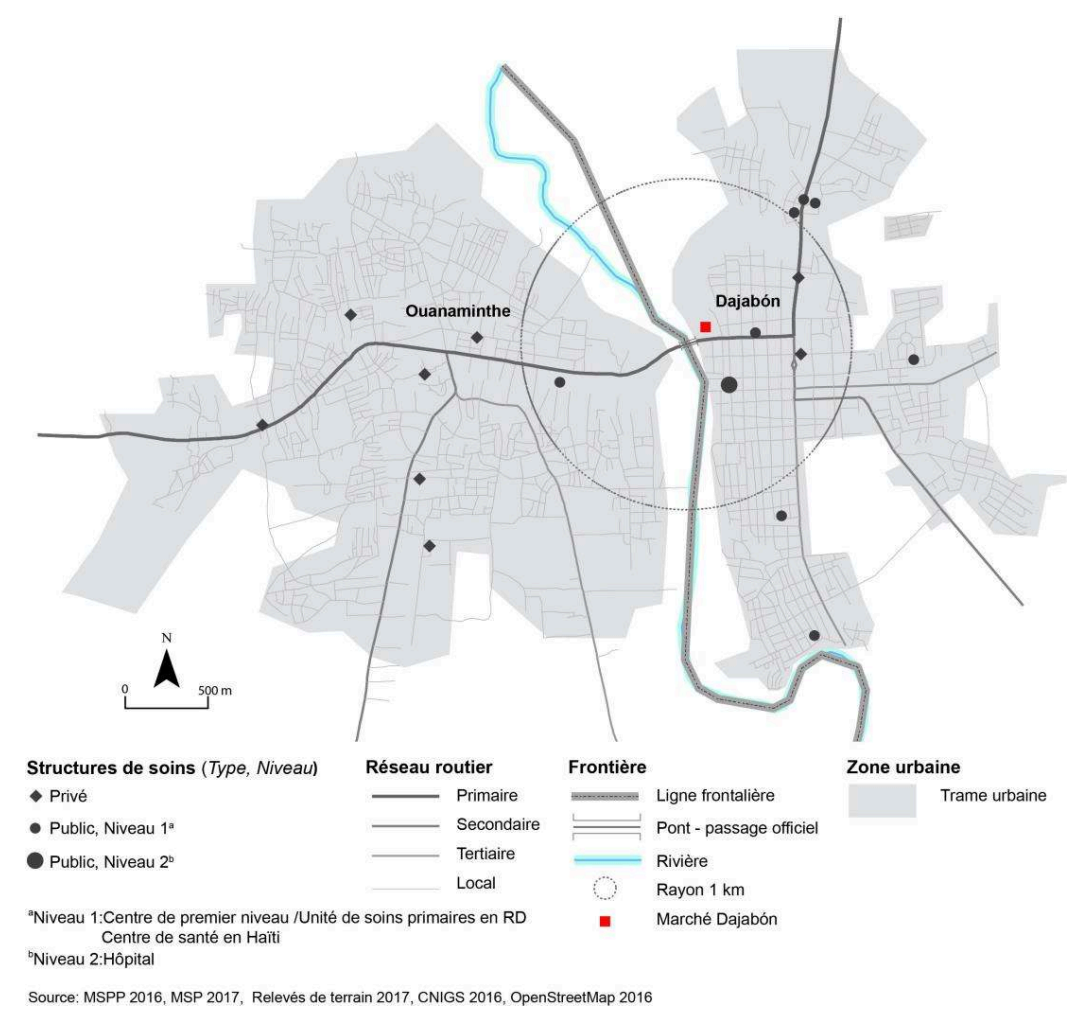

En Haïti, le manque de professionnels de santé est flagrant avec seulement 6,3 professionnels pour 10000 habitants, soit un ratio bien en dessous du seuil minimal suggéré par l'Organisation mondiale de la santé (OMS) (25 pour 10 000). La situation au niveau du département du Nord-Est - dont Ouanaminthe est la ville principale - est loin d'être différente avec un ratio de 6,7 (Ministère de la Santé Publique et de la Population, 2018, p. 44). La République dominicaine compte 28,2 professionnels de santé pour 10000 habitants, un ratio néanmoins bien en dessous de la moyenne régionale des Amériques qui est de 82,6 (World Health Organization, 2018).

De plus, les ressources budgétaires allouées au secteur de la santé en Haïti sont limitées et représentaient moins de 5\% du budget rectificatif national pour 2016-2017 (Institut Haïtien de l'Enfance -IHE/Haiti \& ICF, 2018) contre 12\% en République dominicaine en 2017 (Ministerio de Hacienda, 2017a, 2017b). Le Service national de Santé (SNS) de la République dominicaine estime que l'attention médicale fournie aux étrangers en 2017 (consultation, accouchements, suivi prénatal, les soins fournis dans le cadre des programmes de VIH-SIDA et de Tuberculose) représente un investissement total de 2,98 milliards de pesos dominicains, soit l'équivalent de 55,56 milliers d'euros ${ }^{1}$ (Servicio Nacional de Salud, 2017).

En République dominicaine, le secteur privé est aussi présent (Rathe \& Moliné, 2011). La ville de Dajabón compte un hôpital public de 30 lits (niveau 2) et deux cliniques privées offrant des services spécialisés comme gynéco-obstétrique, médecine interne, services de laboratoire, de radiologie et d'échographie. Tous ces établissements sont proches de la frontière dans un rayon de moins d'un kilomètre), ce qui les rend relativement accessibles aux habitants de Ouanaminthe (figure 6). 


\section{Méthodes}

\section{Méthodes et collecte de données}

23 Cette étude repose sur une approche qualitative descriptive (Sandelowski \& Barroso, 2006). Elle s'appuie sur le recours à plusieurs méthodes combinées à savoir l'observation de terrain et la conduite d'entretiens semi-directifs (Olivier de Sardan, 2008). Les entretiens ont été conduits en mai 2016 et juin 2017. Des entretiens exploratoires informels ont été menés (mai 2016) préalablement afin d'identifier les cliniques privées de Dajabón et établir les contacts avec celles-ci.

\section{Les entretiens avec les patient.e.s}

24 Au cours d'un premier séjour de terrain d'une durée de 15 jours en mai 2016, avec l'aide d'informateurs clés travaillant dans une organisation non gouvernementale (ONG) basée à Ouanaminthe et travaillant sur la frontière haïtiano-dominicaine, 2 personnes ont pu être identifiées pour des entretiens. Au cours d'un deuxième terrain d'une durée de 15 jours en juin 2017, des entretiens auprès d'un corpus de 19 personnes fréquentant les cliniques privées $(n=17)$ et l'hôpital public de Dajabón $(n=2)$ ont été réalisés. Au total, 21 personnes ont été interrogées. Contrairement à ce qui était initialement prévu, en raison des difficultés pour obtenir des références de patient.e.s à travers les réseaux et contacts disponibles à Ouanaminthe, les personnes ont été identifiées en les approchant directement sur les différents lieux de consultation (cliniques privées et hôpital public) avec l'accord préalable des responsables de ces institutions.

Ce changement de stratégie a permis une observation périodique des structures de soins, des fluctuations en termes d'affluence, de clientèle, du temps d'attente, des interactions entre la clientèle et le personnel (médical ou de soutien). Le point d'ancrage étant à Ouanaminthe, il fallait traverser la frontière tous les jours à pied ou en voiture. Ceci a permis de mesurer au quotidien ce que représentait la mobilité pour les patient.e.s en quête de soins de l'autre côté de la frontière. L'ensemble de ces observations étaient consignées dans un journal de terrain. Les difficultés à la frontière, le temps mis pour traverser, les interactions des Haïtiens avec les officiels (militaires ou civils), etc. sont autant d'éléments qui permettent une mise en contexte.

Les entretiens avec les patient.e.s comprenaient les sections suivantes: 1) leur itinéraire thérapeutique; 2) à quel moment, ils ont pris la décision de venir en consultation à Dajabón ; 2) les motifs et la manière dont la décision a été prise ;4) comment ils ont eu connaissance de la clinique ou de l'hôpital ; 5) leurs perceptions de la qualité des soins reçus en Haïti et en République dominicaine ; 6) expérience de la frontière (pratique, difficultés rencontrées, etc.). Les entretiens avec les patients.tes sont menés en créole. Ils ont, dans leur grande majorité, eu lieu sur place (clinique ou hôpital) car, les tentatives pour rejoindre les personnes ciblées une fois qu'elles avaient quitté les structures de soins s'étaient soldées à plusieurs reprises par des échecs (par manque de disponibilité de ces personnes ou dû à des difficultés de communication téléphonique).

Sur l'ensemble des 23 personnes approchées, deux ont refusé. Les 21 autres ont donné leur consentement verbal pour répondre aux questions après avoir pris connaissance de l'objectif de la recherche, mais 19 d'entre elles ont refusé que l'entretien soit 
enregistré. Les entretiens ont été menés par la première auteure et se sont déroulés dans un endroit retiré de la salle d'attente, à l'exception de trois d'entre eux : deux ont eu lieu sur le lieu de travail et un sur le lieu de résidence.

\section{Les entretiens avec les cliniciens et gestionnaires}

Sept entretiens semi-structurés ont également été réalisés auprès de médecins des cliniques privées et hôpital public à Dajabón $(\mathrm{n}=4)$ et des médecins du Centre de santé de Ouanaminthe et du ministère de la Santé publique et de la population d'Haïti $(n=3)$. Sur les sept entretiens, trois ont été enregistrés. Ces personnes ont été contactées directement sur leur lieu de travail. L'objectif de ces entretiens était entre autres de collecter des informations sur les l'organisation du système de santé, les structures de soins et leur fonctionnement, la fréquentation des institutions de santé dominicaines par une clientèle haïtienne, les modalités d'accès aux soins, l'existence de collaboration ou de référence médicale entre Haïti et la République dominicaine. Ces entretiens ont été réalisés en espagnol avec les Dominicain.e.s, en français et/ou en créole avec les Haïtien.ne.s, au gré de leurs préférences et de la dynamique de l'entretien.

\section{Analyse des données}

Pour renforcer la rigueur et la crédibilité des résultats, nous avons adopté un processus d'analyse thématique itératif. Les notes ou les enregistrements des entretiens auprès des patient.e.s, des cliniciens et gestionnaires ont tous été retranscrits dans leur langue originale par la première auteure et traduits lorsque nécessaire ( $\mathrm{du}$ créole ou de l'espagnol vers le français). Le processus d'analyse comprenait la codification manuelle des données, un tableau synthèse en fonction des différentes catégories thématiques définies initialement, un tableau pour établir le profil des patient.e.s, la connaissance de langue, leur expérience de la frontière, leur activité économique. De ce processus, d'autres catégories thématiques ont émergé et des regroupements en sous-catégories ont été effectués.

\section{Considérations éthiques}

Chaque participant a été contacté directement et une description du projet par écrit leur a été fournie. Une fois, la description du projet fournie, leur consentement écrit ou verbal leur a été demandé. Cette recherche a reçu l'approbation du Comité d'Éthique de l'Institut National de la Recherche Scientifique (INRS).

\section{Résultats}

\section{Profil des patientes et patients, des cliniciens et gestionnaires}

Sur les 21 personnes ayant accepté de participer à la recherche, 12 vivent à Ouanaminthe. Les autres habitent dans des villes ou localités voisines : 4 au CapHaïtien, 1 à Fort-Liberté, 1 à Limonade, 1 à Ti Lori, une localité rurale du Centre et 2 n'ont pas donné leur lieu de résidence. La clientèle de ces structures de soins de Dajabón durant la période au cours de laquelle les entretiens ont été effectués était surtout composée de femmes, d'enfants, de personnes âgées et très peu d'hommes. 
Notre corpus le reflète avec: 14 femmes, 1 mère avec un nourrisson, 3 parents avec enfants et 3 hommes dont un âgé.

D'une manière générale, la plupart des personnes venues en consultation dans les deux cliniques ou à l'hôpital étaient accompagnées soit d'un parent ou d'un proche (ami, voisin). Le point de passage frontalier officiel est celui utilisé par l'ensemble des patients et patientes pour se rendre aux établissements de santé. Les contrôles sont variables et dépendent du jour (jour de marché ou pas), du contexte (situation de tensions ou pas) au moment de la traversée, de la pratique de la frontière par les usagers. Le moyen de transport le plus utilisé est la moto ou un taxi-moto.

Les personnes ont presque toutes une pratique récurrente de la frontière; soit elles vont s'approvisionner à Dajabón, soit elles ont une activité économique en lien avec le marché «binational» de Dajabón (commerce, transport de marchandises), soit elles travaillent dans une organisation qui mène des activités sur la problématique frontalière. La majorité des patients et patientes déclarent avoir une activité et donc un revenu.

\section{Itinéraire thérapeutique et mobilité transfrontalière}

\section{Des cheminements pluriels}

Les entretiens ont permis de retracer partiellement l'itinéraire thérapeutique en amont de leur consultation à une clinique ou un hôpital à Dajabón. Les différents récits recueillis décrivent succinctement les structures de soins fréquentés tant en Haïti et en République dominicaine en lien avec leur problème de santé à l'instant t. Parfois, celuici remonte à plusieurs années et a fait l'objet d'un cheminement déjà complexe et sinueux. Pour certains, il s'agit de la première visite, pour d'autres ce sont des visites de suivi médical. Enfin, d'autres viendront en consultation soit pour un problème ponctuel, soit pour un examen.

Le cas d'une femme résidente de Limonade - ville située à environ $52 \mathrm{~km}$ de la frontière - venue en consultation à une clinique de Dajabón pour des douleurs récurrentes illustre bien la sinuosité de l'itinéraire thérapeutique. Ses deux premières visites ont été effectuées dans deux établissements de soins haïtiens distincts (respectivement public et privé), relativement proches de son lieu de résidence et qu'elle a coutume de fréquenter. Après trois ans, elle décide de faire une consultation à clinique de Dajabón, pour retourner ensuite à Trou du Nord (Haïti), le point de départ de son parcours, où elle fait des analyses et une nouvelle consultation.

« La première fois où j'ai ressenti la douleur, j'ai été en consultation à Trou du Nord, on m'a dit que c'était un kyste, un fibrome. C'était en 2014. On m'a donné des médicaments et j'ai suivi le traitement. Depuis trois ans, la douleur persiste. J'ai alors été en 2016 à l'hôpital à Carrefour La mort [du côté haïtien proche de Limonade] [...]. Ils disent n'avoir rien trouvé et m'ont donné des médicaments pour soulager la douleur. La douleur est revenue. Je suis venue à cette clinique [de Dajabon]. C'est la première fois [que je viens à la clinique de Dajabon] [...] J'ai des analyses à faire. Je les ai faites lundi à Trou du Nord [...]» (Femme, clinique privée de Dajabón)

Cumuler, pour un même problème de santé (ou des symptômes s'étalant dans la durée), des consultations auprès de différentes structures de soins, voire combiner médecine traditionnelle et biomédecine, est une pratique courante en Haïti. Les raisons évoquées 
par les personnes concernées pour un tel comportement sont diverses comme la recherche d'une seconde opinion ou l'habitude. "Je vais toujours en consultation dans deux endroits ", dira une patiente pour souligner qu'il s'agit d'une pratique courante chez elle et qu'elle ne se contente pas de l'avis d'un seul médecin. Pour d'autres en revanche, avoir un dossier dans plusieurs cliniques en Haïti même quand un suivi régulier est réalisé chez un médecin à Dajabón est une façon de mettre toutes les chances de leur côté. Il s'agit d'une mesure de prudence en cas d'urgence et d'impossibilité de traverser la frontière, d'un comportement judicieux selon les propos d'une personne du corpus. Un monsieur signale avoir amené sa femme suivie dans une clinique privée à Dajabon en consultation une fois au Centre de santé de Ouanaminthe et aussi une fois à l'hôpital de Fort-Liberté, au cas où un problème surviendrait en pleine nuit. Il s'agit, selon lui, d'une précaution pour parer à toute éventualité face aux aléas de la frontière.

Il existe néanmoins des cas de «rupture » complète avec le système de santé haïtien comme cette patiente qui, après une mauvaise expérience lors d'une hospitalisation pour un épisode d'hypertension et d'accident vasculaire cérébral dans une clinique privée de Ouanaminthe, est hospitalisée plusieurs jours dans une clinique privée de Dajabón où elle effectue depuis son suivi médical.

«Je suis venue [...] au mois de mai 2016. Depuis, je ne vais plus en consultation en Haïti. Aujourd'hui, je suis venue pour mon rendez-vous de suivi [...] Je viens chaque deux ou trois jours [pour contrôler la tension artérielle] » (Femme, clinique privée de Dajabón).

Enfin, certains des médecins dominicains de cliniques privées de Dajabón rencontrés signalent qu'ils réfèrent parfois leurs patientes à l'hôpital de Dajabón, notamment quand celles-ci ont des difficultés économiques et qu'une intervention chirurgicale doit être envisagée. La référence à l'hôpital public de Dajabón peut, lorsque la complexité du cas le requiert, aboutir à une référence à un hôpital du troisième niveau, à Santiago qui se trouve à $135 \mathrm{~km}$ à l'est de la ville de Dajabón. Des évidences pour un tel type d'itinéraire n'ont pas pu être collectées sur le terrain. Néanmoins, une personne rencontrée à une clinique privée de Dajabón indique y avoir été référée par une clinique privée de Ouanaminthe pour une échographie. Le médecin de la clinique de Dajabón lui indique qu'elle doit se rendre à l'hôpital de Dajabón pour son traitement. Le personnel de l'hôpital de Dajabón refuse la traiter et lui explique qu'elle doit retourner en Haïti pour son traitement. Cette femme retourne voir le médecin de la clinique de Dajabón pour obtenir de ce dernier une référence qui lui permettrait d'être admise et traitée à l'hôpital de Dajabón. Ainsi, cette patiente s'est retrouvée à faire des allersretours à Dajabón entre une clinique privée et un hôpital public pour recevoir finalement un traitement. Ce cas illustre les difficultés auxquelles font parfois face les Haïtien.ne.s pour obtenir des soins en République dominicaine.

Force est de constater par ailleurs que, peu de personnes déclarent avoir fréquenté le centre de santé de Ouanaminthe. Les motifs d'un tel évitement sont divers comme on le verra plus loin. Les personnes rencontrées ont plutôt tendance soit à consulter des cliniques privées en Haïti (Ouanaminthe et villes avoisinantes), à se rendre directement dans des hôpitaux « réputés » de la région (Trou du Nord, Milot, parfois Fort-Liberté) pour ensuite, selon le cas se rendre dans une clinique privée de Dajabón ou à l'hôpital public de cette ville. La rupture avec les structures de soins en Haïti ne semble pas définitive. 
40 Au regard de ces mobilités thérapeutiques individuelles reliant deux systèmes de santé originellement disjoints, il convient de s'interroger sur le rôle de la frontière dans cette mobilité d'une part et de cerner d'autre part, comment l'obstacle qu'elle pourrait représenter est surmonté.

\section{La gestion de la frontière : un élément déterminant de la mobilité thérapeutique}

41 La durée des trajets pour se rendre à Dajabón ne semble pas constituer un obstacle à la mobilité thérapeutique des patients et patientes. En effet, plusieurs des personnes rencontrées n'habitent pas à proximité de la frontière. Plusieurs d'entre elles font un trajet d'une heure et demie à deux heures, voire de trois heures, pour une visite médicale d'environ 30 minutes à Dajabón, ou pour simplement récupérer des résultats d'analyses médicales. Cela ne tient pas compte du temps requis pour traverser la frontière. Celui-ci est variable, allant de 15 minutes environ en temps normal à une heure, voire plus les jours de marché selon l'affluence. D'autres facteurs conjoncturels (conflits, augmentation du niveau de contrôle du côté dominicain, etc.) peuvent causer des délais dans le passage de la frontière.

42 Une femme habitant à Limonade, une ville située à environ $50 \mathrm{~km}$ de la frontière, raconte avoir quitté sa résidence à $6 \mathrm{~h}$ du matin pour arriver à la clinique à $8 \mathrm{~h} 30$, après avoir passé une heure à la frontière à attendre l'ouverture de la barrière à $8 \mathrm{~h}$. Une autre indique avoir fait trois heures à moto pour sortir de Tilori, une localité rurale haïtienne située à une cinquantaine de kilomètres au sud de Ouanaminthe, et se rendre en consultation à Dajabón. Pour elle, cependant, Dajabón représente une option plus avantageuse et accessible physiquement que l'hôpital de Hinche (Haïti) situé à environ $64 \mathrm{~km}$ de son lieu de résidence. Et ceci, en tenant compte des aléas en lien avec l'offre du service du côté haïtien (spécialiste absent, équipement en panne, etc.), venir en consultation, faire des analyses ou chercher des résultats peut impliquer de mobiliser une journée complète. Ainsi, certaines personnes déclarent profiter de leur déplacement pour s'approvisionner à Dajabón ou régler certaines choses en lien avec leurs activités professionnelles ou personnelles. D'autres viendront spécifiquement pour leur visite médicale.

43 Mais, dans certains cas, le déplacement peut être de longue durée (plusieurs jours), notamment dans le cas d'une hospitalisation. Une patiente raconte avoir été hospitalisée près d'une dizaine de jours à une clinique privée de Dajabón. Une telle situation entraine des déplacements continus (des allers-retours quotidiens) pour les membres de sa famille associés à la gestion de la thérapie.

«J'ai été hospitalisée pendant neuf jours. Ma cousine est restée avec moi durant mon hospitalisation. Chaque jour, Maman m'envoyait de la nourriture » (Femme, Clinique privée de Dajabón).

44 Cependant, il faut souligner que la gestion de la frontière par les autorités tend à contraindre la mobilité et à moduler, à certains égards, la dimension temporelle de celle-ci. Les déplacements à travers le point de passage officiel sont limités dans le temps, soit la durée d'ouverture journalière de la frontière qui est de 9 heures (du lundi au samedi de 8 à $17 \mathrm{~h}$ ) et de 7 heures (dimanche de 9 à $16 \mathrm{~h}$ ). La fermeture de la frontière est parfois imprévisible; elle dépend des conjonctures nationale et locale. Ainsi, l'ouverture peut être décalée de plusieurs heures ou un conflit peut occasionner sa fermeture anticipée. Durant les observations de terrain en juin 2017, l'ouverture de la frontière a été décalée une fois d'environ trois heures avec une forte présence 
militaire du côté dominicain. Les raisons de cette fermeture temporaire sont méconnues et à notre connaissance, aucune communication officielle n'a été diffusée. Les contrôles frontaliers, notamment du côté dominicain, sont cependant plus importants, et ceci, depuis 2 à 5 ans environ, selon les médecins et gestionnaires interrogés. Les jours de marché, les lundis et vendredis, les flux à la frontière sont particulièrement denses (plus de 30 mille personnes, selon des estimations non officielles, font des allers-retours). Ceci complique la circulation piétonne et motorisée et de ce fait, le passage. Contrairement aux autres jours, les Haïtiens et les Haïtiennes peuvent traverser «librement" la frontière les jours de marché, mais les "gardes dominicains » (en uniforme ou en civil) sont omniprésents particulièrement dans les limites du périmètre du marché ; ils surveillent ceux qui tenteraient d'aller au-delà. La tension est vive. Ces journées sont ponctuées de conflits divers et de fouilles effectuées par les militaires dominicains sur des vendeurs et vendeuses d'origine haïtienne se terminant soit par des exactions ou la confiscation des marchandises. Certaines des personnes rencontrées ont d'ailleurs déclaré éviter de venir en consultation à Dajabón les jours de marché; il y a trop d'encombrements ("Jou mache yo gen twòp ankombreman ») déclare une femme venue du Cap-Haïtien pour une consultation à Dajabón.

Ces contrôles tendraient à affecter les flux selon certains. Un médecin dominicain d'une clinique privée de Dajabón dont une part relativement significative de la clientèle est haïtienne signale avoir constaté depuis cinq ans une baisse dans le flux de patients haïtiens qu'il explique par ce durcissement à la frontière. Une opinion que partage un responsable du MSPP.

«Maintenant, il y a un ralentissement dans le flux des gens qui traversent vers la frontière pour aller en République dominicaine, mais, eh... eh... parce que bon... il y a cette tension qui existe depuis tantôt deux ans entre Haïti et la République dominicaine. Mais, dans le temps, les gens pour un oui ou pour un non traversaient pour aller chercher des soins en République dominicaine » (Responsable MSPP).

Mais les avis sont partagés en ce qui trait à l'évolution de l'affluence de patient.e.s d'origine haïtienne. En effet, un médecin dominicain travaillant dans une autre clinique privée estime en revanche qu'il y aurait eu, au cours des dernières années, une hausse du nombre de patient.e.s d'origine haïtienne imputable à une amélioration des relations haïtiano-dominicaines.

«L'amélioration des relations haïtiano-dominicaines favorise une augmentation des entrées de ressortissants haïtiens qui viennent pour des soins médicaux ici, non seulement en gynécologie, mais dans tous les domaines » (Médecin, Clinique privée de Dajabón).

47 Ces points de vue reflètent les différences de perception existant au sein des sociétés haïtiennes et dominicaines quant aux relations entre les deux pays. Il convient de souligner que celles-ci, au cours des quinze dernières années, ont connu des moments de tension ${ }^{2}$ parallèlement au développement d'une coopération transfrontalière encore embryonnaire (projets financés par des organisations internationales ou des initiatives d'acteurs locaux divers).

Traverser la frontière est généralement compliqué pour les Haïtien.ne.s. Toutefois, certaines personnes, incluant celles ne disposant pas de papiers (passeport ou visa), affirment ne pas rencontrer de difficulté majeure pour traverser. Pour un médecin dominicain, la majorité des gens qui traversent la frontière ont des documents légaux et pourtant, ils n'échappent pas aux pressions. Selon ce médecin, il y aurait des gens 
intéressés à venir, mais qui y renoncent. Les récits de plusieurs personnes montrent en effet que des négociations sont souvent nécessaires. De l'argent est parfois soutiré à certains.

«Quand on traversait, on ne voulait pas nous laisser passer. Nous avons dit que nous allions à l'hôpital et on nous a laissé rentrer. Nous avons payé 100 pesos $[1,86 €]$ plus le prix du transport » (Femme, clinique privée de Dajabón).

En fait, il s'agit d'une dynamique à géométrie variable dont la lecture est parfois difficile. Elle donne lieu à un sentiment d'injustice comme en témoignent les propos ciaprès.

«Il n'est pas question de personne en situation de légalité ou d'illégalité, le Dominicain, ne respecte personne [...] là où un délinquant peut être à l'aise pour rentrer, toi qui as des papiers, tu ne l'es pas. Parce que toi qui as des papiers, les gens te tiraillent ici et là, ils te dupent, te font perdre du temps, te disent des choses désagréables, tu dois faire plein de déclarations pour passer bien que tu aies un passeport, que tu aies un visa et que tu aies passé l'immigration. Et le délinquant [...] c'est lui l'ami des chefs, il passe comme il veut, quand il veut, là où il veut " (Homme, parlant de son expérience avec sa femme).

Ceci montre les difficultés auxquelles sont confrontées les Haïtien.ne.s pour passer la frontière et révèle par ailleurs le décalage dans les niveaux contrôles exercés ainsi que l'existence de réseaux favorisant le développement de trafics ou pratiques frauduleuses. Ainsi, dans un contexte où légalité et corruption s'entremêlent, où la migration demeure une épineuse question, les populations locales mobilisent compétences et réseaux sociaux pour se rendre à Dajabón et tenter de trouver une solution à leurs problèmes de santé.

\section{Mobilisation du réseau social et de compétences diverses}

L'analyse des entretiens montre que des compétences diverses doivent être mobilisées par les patient.e.s et leurs proches pour traverser la frontière.

Premièrement, les personnes rencontrées viennent rarement seules en consultation. Les enfants et les personnes âgées sont évidemment accompagnés de leurs parents (père, mère, enfants). Les femmes, quant à elles, s'organisent pour venir en groupe (avec des amies ou des connaissances) ou accompagnées de leur conjoint ou de leur mère. S'il est difficile, compte tenu du nombre réduit de personnes rencontrées d'en déduire un modèle de comportement, on peut faire l'hypothèse que des précautions semblent être prises pour traverser la frontière. Autrement dit, pouvoir mobiliser des contacts, des connaissances (Haïtiens ou Dominicains) semble constituer un atout pour traverser la frontière et se faire soigner en République dominicaine. Une femme est venue dans la voiture d'un ami. Une autre accompagnant son fils pour qu'on lui mette un plâtre a été conduite à moto par un voisin de nationalité dominicaine. Habitant une communauté rurale située dans le Département du Centre en Haïti (plus au sud de Dajabón), à proximité de la frontière, elle indique avoir fait un voyage d'environ 4 heures avec son fils.

Deuxièmement, le choix de consulter à Dajabón repose rarement sur une connaissance fine ou préalable du système de santé dominicain. Sur l'ensemble des personnes, seules deux ont déclaré avoir vécu pendant un temps en République dominicaine. Par conséquent, les décisions semblent plutôt résulter du "bouche-à-oreille ", entre autres du partage par des tiers (amis, connaissance) d'expériences positives, de la 
connaissance par un proche du système de santé dominicain ou d'informations provenant de personnes travaillant dans le secteur. Un monsieur raconte que c'est au cours d'une conversation entre amis qu'ils, lui et sa femme, ont eu des informations sur une clinique privée de Dajabón.

Troisièmement, l'expérience de la frontière constitue un atout non négligeable à deux niveaux : i) des contacts et une pratique pouvant faciliter le passage de la frontière ; ii) une connaissance même rudimentaire de la langue. En effet, le vécu de la frontière permet d'être (re)connu des "gardes dominicains ", avoir un savoir-faire et savoir sur quelle ficelle tirer en cas de difficulté.

"Je n'ai pas de problème pour traverser, je vends ici tous les jours. Je vends au marché avec un dominicain. Je n'ai pas eu de problème pour traverser avec l'enfant parce qu'on me connait, mais ces jours-ci, les enfants ne traversent pas » (Femme, accompagnant son enfant, Hôpital de Dajabón).

55 Ces propos montrent comment cette femme, grâce à sa pratique de la frontière, a pu contourner les dispositifs de surveillance mis en place pour limiter le trafic d'enfants. En principe, un adulte accompagné d'un mineur ne peut pas traverser sans autorisation. Il est intéressant de souligner par ailleurs que sur l'ensemble des personnes rencontrées, la grande majorité comptait avec une expérience de la frontière, souvent en lien avec le marché de Dajabón. "Je travaille sur la frontière » dira l'une des personnes. Une autre indique être venue avec quelqu'un travaillant à l'une des cliniques et dit: "N'était-ce de cette personne, nous n'aurions pas pu rentrer. Nous avons payé 200 gourdes [2,78 €] pour rentrer ». Ainsi, les moins habitués mobilisent des contacts avec lesquels ils traversent ou qui peuvent les aider à surmonter les obstacles auxquels ils sont confrontés lors de leur passage. Les récits montrent donc l'importance à la fois du savoir-traverser acquis avec la pratique de la frontière (capital spatial) et du réseau social (capital social) dans la mobilité transfrontalière.

Quatrièmement, la connaissance de l'espagnol est un avantage pouvant s'avérer utile dans les négociations à la frontière. Mais, sa méconnaissance n'est pas vécue comme une contrainte. En effet, celles et ceux qui déclarent parler espagnol sont peu nombreux $(n=3)$ alors que plusieurs disent comprendre l'espagnol sans pour autant le parler (soit une maîtrise incomplète de la langue). Un cas probant est celui d'une femme ne parlant pas l'espagnol qui est accompagnée de sa mère qui, elle, d'après ses dires, "se défendait». Ainsi donc, l'accompagnateur (connaissance ou membre de la famille) peut servir d'interprète le cas échéant.

57 Compte tenu ces contraintes, qu'est-ce qui motive les personnes interrogées à aller chercher des soins à Dajabón?

\section{Le recours transfrontalier aux soins : pourquoi traverser?}

\section{Référence médicale ou « bouche-à-oreille »}

La référence médicale, soit l'orientation d'une structure de soins publics haïtienne vers une dominicaine, n'existe pas formellement entre Haïti et la République dominicaine. C'est l'avis des médecins dominicains et haïtiens rencontrés. Le protocole au sein du système de santé publique haïtien ne permet, selon un médecin haïtien, de référer un patient à l'étranger. Ceci n'empêche qu'un médecin peut, si le patient désire se faire soigner dans un autre pays, préparer un rapport avec les informations indispensables pour le suivi ou la réalisation d'examens plus poussés. Cela étant, les références 
médicales institutionnelles ou formelles sont rares aux dires des médecins dominicains. Les «références sociales" - équivalent au «bouche-à-oreille»- sont bien plus courantes comme l'indique ce médecin dominicain d'une clinique privée de Dajabón :

«Les références sont plutôt sociales, l'un dit à l'autre. Il y a quelques références médicales, mais peu nombreuses. La référence est informelle surtout pour l'échographie. 90\% des femmes viennent au début de leur grossesse » (Médecin, clinique privée de Dajabón).

Un responsable du MSPP, tout en reconnaissant l'absence d'un système de référence formelle entre les deux pays, souligne que la pratique peut être conditionnée par des urgences médicales, notamment des cas demandant une prise en charge immédiate et un traitement spécialisé.

« Non, on ne fait pas de références, [...] parfois on est obligé d'écrire les notes de référence pour la République dominicaine [...] on n'a pas un système de référence formel avec la République dominicaine. Mais, [...] on sait qu'il y a déjà des cas [...] que de toute façon il faut les référer vers la République dominicaine. Par exemple, s'il y a un accident vasculaire cérébral, on réfère la personne directement en République dominicaine parce qu'on sait qu'il n'y aura pas une bonne prise en charge de notre côté à moins d'envoyer la personne jusqu'à Port-au-Prince à [l'hôpital] Bernard Mevs. Mais, sinon en République dominicaine à deux heures de la frontière, la personne sera déjà dans un hôpital de Santiago où on peut faire une bonne prise en charge [...]» (Responsable MSPP).

Mais, prendre la décision de se faire soigner à Dajabón semble, le plus souvent, être une décision individuelle ou familiale (patient.e ou ses proches). Les récits des patient.e.s mettent en lumière le rôle des proches dans la gestion de la thérapie et l'influence subséquente sur l'itinéraire thérapeutique. Plusieurs facteurs interviennent dans la prise de décision dont l'expérience positive de proches, les informations obtenues d'amis ou de connaissance.

61 Une dame déclare par exemple avoir été amenée en consultation à Dajabón par son mari ; ce dernier, ayant vécu en République dominicaine, connaissait la clinique. Une autre personne raconte comment ses parents ont pris la décision de l'amener à Dajabón alors qu'elle était hospitalisée à une clinique privée de Ouanaminthe : «Ils voulaient me garder, mais ma mère et mon père ont pris la décision de m'amener ici [...] ma mère vient aussi en consultation ici». De plus, les informations obtenues et les expériences partagées peuvent porter le patient à choisir une clinique plutôt qu'une autre. Une femme indique que c'est une « sèvant» (servante, personne fréquentant la même église qu'elle) qui l'a référée à une clinique spécifique de Dajabón ; une autre déclare que sa voisine l'a mise en contact avec quelqu'un travaillant à l'une des cliniques de Dajabón. En définitive, les informations circulent à travers différents canaux, différents réseaux. Un monsieur raconte l'expérience de son père qui, quoiqu'ancienne (entre 2005 et 2007), illustre bien la diversité des sources d'informations dans le recours transfrontalier aux soins.

«On a eu l'information qu'un groupe de spécialistes dominicaines allait opérer gratuitement [des patients souffrant de hernie] à l'hôpital de Dajabón [...] c'est à l'Église que l'annonce a été faite [...] une liste a été dressée par les responsables de l'Église. Mon père est parti avec eux [...] il n'a eu aucune formalité à remplir ».

62 Force est de constater qu'en amont du choix, l'évaluation des résultats obtenus au cours des étapes antérieures de l'itinéraire par le patient et ses proches pèse dans la prise de décision. Beaucoup des témoignages recueillis révèlent en effet que la 
recherche de résultats (traitement efficace, guérison, etc.) est au centre des préoccupations des patients et patientes.

\section{La quête de résultats}

L'itinéraire thérapeutique, comme l'indiquent la plupart des récits, commence en Haïti. La décision de se rendre à Dajabón découlerait plutôt d'une mauvaise expérience (en lien avec le diagnostic ou la thérapie) ou de l'absence de résultats à l'issue du traitement reçu. Les médecins dominicains interrogés estiment que les cliniques de Dajabón seraient le dernier recours après un long itinéraire thérapeutique avec peu ou sans résultats dans les cliniques/hôpitaux en Haïti.

« Beaucoup d'entre eux viennent parce [...] qu'ils ont été traités et n'ont pas eu [ou] vu d'amélioration. Par conséquent, ils cherchent une autre [...] option, une autre opinion » (Médecin, clinique privée Dajabón).

Le récit de plusieurs patients souligne effectivement ce long périple, cette quête de résultats et comment les cliniques à Dajabón apparaissent comme «la» solution. Au regard de leurs déclarations quant aux raisons d'une telle décision, "rezilta» (résultats) est un leitmotiv $(\mathrm{n}=10)$. En guise d'exemple, le récit d'un couple qui, après plusieurs tentatives non concluantes à Ouanaminthe, à Fort-Liberté et au Cap-Haïtien (à près de $65 \mathrm{~km}$ au nord-ouest de Ouanaminthe), choisit de se rendre en consultation à Dajabón.

«[...] nous avons eu 4 expériences déjà ici, à Ouanaminthe, à Fort-Liberté et au CapHaïtien, nous n'avons malheureusement pas obtenu le résultat que nous désirions. C'est pour cela que nous étions toujours à la recherche d'un endroit qui pourrait vraiment nous aider [...] Et... nous n'étions pas vraiment optimistes quand nous sommes allés parce que nous avions déjà vu un spécialiste dans le nord qui nous avait été recommandé aussi et qui avait beaucoup de résultats, beaucoup de personnes parlaient de lui, et nous aussi sommes allés là, mais nous n'avons vraiment pas trouvé le résultat souhaité [...]. Alors, nous sommes allés là [une clinique privée de Dajabón] » (Homme, clinique privée de Dajabón).

Mais, les traitements proposés ne sont pas toujours acceptés ou le sont avec une certaine réserve. Deux cas l'illustrent. Le premier est celui d'une femme qui décide d'aller à une clinique de Dajabón pour des douleurs et démangeaisons au bras que les médicaments prescrits par son médecin traitant à Ouanaminthe ne soulagent pas. Son bras avait été plâtré à une clinique privée Ouanaminthe après un accident de motocyclette. À Dajabón, on lui propose de lui «casser à nouveau le bras »; elle décide alors de se rendre à l'hôpital de Milot, puis de faire la réhabilitation dans un centre privé à Ouanaminthe.

Le deuxième est celui d'une femme qui a été opérée à une clinique privée de Dajabón. Selon elle, le médecin en Haïti aurait eu une approche différente : «Le dominicain a enlevé l'utérus, alors que le médecin en Haiti aurait essayé de faire en sorte de le garder ". Mais, sa peur due, entre autres aux difficultés pour obtenir du sang si elle était opérée en Haïti, l'a incitée à privilégier l'option dominicaine. Elle continue d'ailleurs à faire son suivi à Dajabón. Elle met ainsi l'accent sur un autre motif souvent évoqué par plusieurs personnes, la "faiblesse» des services (disponibilité de ressources, d'équipements, d'intrants, etc.) comme facteur intervenant dans leur décision. 


\section{Carence de services, manque de ressources, temps d'attente}

67 Une femme déclare d'entrée de jeu quand la question lui est posée sur les motifs de sa présence : «En Haïti, il n'y a pas d'hôpitaux, il n'y a pas de services [...], pas de services de qualité », un constat sans appel basé sur une mauvaise expérience, résultant en une aggravation de son état de santé dans une clinique privée de Ouanaminthe.

68 D'autres font valoir le manque de ressources techniques: "la difficulté pour faire des analyses, une échographie», "tu ne peux pas faire une échographie, car l'appareil chauffe». C'est aussi l'absence des médecins, de techniciens ou tout simplement de services spécialisés qui est mise de l'avant. Une personne âgée habitant Fort-Liberté venue en consultation dans une clinique privée de Dajabón explique :

« Fort-Liberté a un hôpital, mais, il n'est pas bien structuré, il lui manque beaucoup de service de base. J'ai besoin d'un cardiologue ou d'un urologue. Mais il n'y a pas ces services à Fort-Liberté » (Homme, clinique privée Dajabón).

69 Pourtant, le centre de santé de Ouanaminthe - situé à $20 \mathrm{~km}$ de Fort-Liberté - compte un urologue selon les informations recueillies auprès d'un responsable dudit centre. La méconnaissance de cette offre de soins spécialisés résulte peut-être du manque de communication sur les services offerts et disponibles dans la région. Mais, d'une manière générale les personnes interrogées au cours de l'enquête sont peu enclines à fréquenter les structures de soins étatiques, en particulier le centre de santé de Ouanaminthe pour plusieurs des raisons évoquées plus haut, mais aussi à cause de la perte de temps. Le temps d'attente parait être, au regard des propos recueillis, un problème constaté au niveau de divers établissements publics de soins de la zone. « $T u$ peux passer la journée et ne jamais recevoir de soins » déclare une femme concernant par exemple l'hôpital public au Cap-Haïtien.

70 Le temps d'attente serait particulièrement long au centre de santé de Ouanaminthe, voire à certaines cliniques privées de Ouanaminthe qui sont très fréquentées, selon plusieurs personnes. "Au centre de santé, il faut avoir du temps" dit une patiente rencontrée à Dajabón. Pourtant, au regard des estimations effectuées, les patient.e.s doivent mobiliser plusieurs heures (une demi-journée environ) pour une consultation dans une clinique privée à Dajabón. Dans certains cas, ils arrivent à la clinique dès $8 \mathrm{~h}$ et n'arrivent à voir le médecin qu'aux environs de midi. Selon les informations recueillies et nos observations, les médecins n'arrivent généralement pas avant midi, soit après leur travail à l'Hôpital de Dajabón. En cas d'urgence, ils peuvent toutefois être appelés et arriver avant. Cette attente ne semble pourtant pas déranger ceux qui fréquentent les cliniques privées de Dajabón.

\section{Pour des relations soigné.e/soignant.e empathiques}

71 La relation soigné.e/soignant.e apparaît comme une des pierres d'achoppement. Elle influence le degré de satisfaction ou d'insatisfaction observée chez les personnes interrogées. Dans leur récit, les patient.e.s, quand il leur est demandé, de comparer les services reçus en Haïti avec ceux reçus en République dominicaine (Dajabón ou Santiago, que ce soit dans le privé ou le public) ont une appréciation largement plus favorable de l'accueil qui leur est fait en République dominicaine.

72 Plusieurs personnes ont émis des réserves quant au suivi réalisé par les médecins haïtiens consultés. Elles ont eu à insister sur leur manque de disponibilité en dehors des heures de consultation, et ce, même en cas d'urgence. Une femme commente: «En 
Haïti, le médecin est introuvable en dehors de ses heures de travail. Il est difficile de le trouver même quand il y a une urgence ". Sans vouloir généraliser, une personne rencontrée estime qu'il y aurait moins de négligence et que le suivi serait plus serré chez son médecin à Dajabón comparativement à ses expériences antérieures en Haïti.

«Le Dominicain ne fait pas de négligences. [...] il arrive que nous ayons un rendezvous à telle date, tu as un problème [...] Il est 10h, ton téléphone sonne, ils te font appeler, aujourd'hui tu avais rendez-vous et tu n'es pas venu. Cela montre qu'ils sont toujours en train de suivre le patient de près. Automatiquement qu'un problème se présente, ils ont le temps de l'identifier. Mais ici [Haïti], tu vas quand tu veux [...]» (Homme, clinique privée Dajabón).

Le manque d'empathie chez les professionnels de santé en Haïti est soulevé par la plupart des personnes rencontrées : "on ne soucie pas de vous ", "vous n'êtes pas pris en considération ", «le médecin n'a pas vraiment de patience pour parler au patient. Il ne lui pose pas de question». Or, la communication soigné.e/soignant.e est une composante essentielle de la pratique clinique. L'accueil constitue une autre cause d'insatisfaction des patients et patientes fréquentant les structures de santé haïtiennes, en particulier celles qui sont publiques. Les responsables du Centre de Santé de Ouanaminthe sont conscients des failles à ce niveau. Selon, eux il y a un problème de formation au niveau du personnel et des efforts devraient être faits en ce sens.

«L'accueil est un facteur important. Il y a un gros problème de formation continue.

Les conditions de travail des employés dans l'état ne sont pas faciles" (Gestionnaire)

74 En revanche, aucune des personnes interrogées que ce soit dans les cliniques privées ou à l'hôpital de Dajabón ne s'est plainte de leurs interactions avec les professionnels de santé dominicains. "Les gens m'ont bien accueilli à la clinique. Le service est meilleur ». Mais, lors de nos observations, une certaine indifférence de la part du personnel de santé à l'hôpital public de Dajabón à l'égard des Haïtien.ne.s sollicitant des informations a été constatée. Minn (2004) a d'ailleurs relevé un comportement différencié à l'égard des Haïtien.ne.s à l'hôpital de Dajabón, les relations étant plus brusques, voire empreints de préjugés notamment envers ceux ou celles à plus faibles revenus. Les temps d'attente des Haïtien.ne.s peuvent, par exemple, être beaucoup plus longs que ceux des Dominicain.e.s. Les allers-retours forcés des patient.e.s entre les structures de soins privées et publiques dominicaines, comme relaté plus haut, soulignent aussi des différences dans l'attention et l'accueil réservé aux patient.e.s d'origine haïtienne.

\section{Des mécanismes mis en place pour faciliter la communication}

Contre toutes attentes, la barrière linguistique ne semble pas constituer un handicap dans les interactions entre les patient.e.s qui ne maitrisent pas la langue espagnole et les professionnels de santé. Un interprète est mis à la disposition de leur clientèle haïtienne par les cliniques privées de Dajabón pour faciliter la communication lors des consultations. Cette intermédiation linguistique semble toutefois ne soulever aucun problème majeur aux patient.e.s, ni aux médecins qui semble s'en accommoder. Une certaine relation de confiance existerait entre interprète/patient.e et médecin/ interprète.

Deux éléments semblent entrer en ligne de compte dans la relation patient.e/ interprète: habitude et expérience. L'habitude permet l'établissement de rapports privilégiés et la création d'un climat de confiance. La longévité, quant à elle, donne lieu 
à une expertise et des compétences appréciées par les patient.e.s. Ceci contribue à mettre en confiance les patient.e.s qui peuvent alors parler ouvertement au médecin sans se soucier de la barrière linguistique. Celle-ci n'est pas sans risques comme le souligne un des médecins dominicains :

« Par exemple, il y a ici un jeune homme qui est interprète [...] Il est haïtien et parle très bien la langue. Mais... je le paie parce que je comprends que je ne peux pas avoir une patiente, lui parler et ce qu'elle me répond « oui, oui, oui ». En plus, j'ai eu des complications avec une patiente parce qu'elle ne comprenait pas comment utiliser un médicament. J'ai eu une Haïtienne qui a pris un ovule [...] Oui un ovule, un ovule, quelque chose qui va dans le vagin... elle l'a avalé malgré sa prescription lisible [...] puisqu'imprimée de l'ordinateur. Mais, elle n'a pas compris que c'était pour là. La barrière de langue est parfois dangereuse en médecine " (Médecin, clinique privée Dajabón).

En l'absence d'interprète, les patient.e.s, s'organisent aussi. S'ils ne sont pas accompagnés d'une personne parlant/comprenant l'espagnol, ils sollicitent l'appui de patient.e.s ou de tiers le cas échéant. Une femme venue seule pour une échographie à l'hôpital de Dajabón et ne parlant, ni ne comprenant l'espagnol, a dû demander de l'aide dans la salle d'attente pour communiquer et s'orienter. Les techniciens responsables de l'examen, ne s'accordant pas sur le sens d'une information fournie par la patiente, ont cherché à la vérifier avec une personne, en l'occurrence la première auteure de l'article assise dans la salle d'attente et qui a fait office d'interprète.

\section{Un investissement}

Les patient.e.s paient eux-mêmes pour les services de santé (consultation, examen, achat de médicaments) qu'ils contractent à Dajabón en particulier dans les cliniques privées. Les coûts y sont légèrement plus élevés que ceux pratiqués en Haïti ce, notamment à cause du taux de change gourde/peso dominicain peu favorable aux Haïtien.ne.s.

Les frais de consultation varient d'une clinique privée à l'autre en Haïti : de 750 gourdes $(9,90 €)$ à 1500 gourdes $(19,82 €)$ contre l'équivalent de 1320 gourdes (1000 pesos dominicains), soit $18,78 €$, à une clinique privée de Dajabón. En revanche, une consultation au centre de santé de Ouanaminthe coûte 50 gourdes $(0,66 €)$ et une échographie 300 gourdes $(3,96 €)$.

Certaines des personnes rencontrées mentionnent les problèmes d'accessibilité financière aux soins de santé auxquels elles sont confrontées. Les contraintes économiques sont d'autant plus grandes que les revenus des patient.e.s sont faibles et de ce fait, les moyens financiers mobilisables limités. Pour se faire une idée, il convient de signaler que le revenu d'activité moyen mensuel en Haïti s'élevait en 2012 à 4830 gourdes, soit $86,85 €$ (Herrera Javier et al., 2014). Le salaire minimum officiel de 300 gourdes $(5,39 €)$ par jour $(2012)$ a été relevé à $500(5,65 €)$ gourdes en 2018 , alors que, parallèlement, la gourde s'est considérablement dépréciée durant cette période. $\mathrm{Au}$ regard de ces chiffres, il ressort que les dépenses de santé représentent une part significative du budget du ménage quand ce dernier doit y faire face; elles peuvent, le cas échéant augmenter la vulnérabilité des ménages les plus pauvres.

«Les services sont excessivement chers. L'écart dans le change peso/gourde est un problème [...] Chaque fois que je viens pour une consultation, je paie 1000 pesos $[18,65 €]$. Pour récupérer des résultats, je dois payer 500 pesos $[9,32 €]$. Si huit jours s'écoulent avant que tu ne viennes chercher les résultats, tu dois alors payer 1000 
pesos $[18,65 €]$. Je viens en consultations chaque mois et je dois débourser 1000 pesos $[18,65 €]$. Depuis trois mois, je viens en consultation deux ou trois jours par semaine. J'ai dû dépenser 7400 pesos $[138,01 €]$ pour m'acheter des médicaments » (Femme, clinique privée Dajabón).

81 Les propos de cette patiente montrent clairement l'effort financier consenti pour résoudre ses problèmes de santé et se faire soigner à Dajabón. Celui-ci est d'ailleurs reconnu par les médecins des cliniques privées de Dajabón qui soulignent le sérieux des Haïtien.ne.s quant au paiement de leurs dettes.

D’un autre côté, les Haïtien.ne.s rencontré.e.s à l'Hôpital de Dajabón disent avoir payé pour les soins qui leur ont été prodigués. En effet, une des patientes rencontrée et venue se faire soigner pour un problème orthopédique indique avoir payé 12000 pesos $(223,70 €)$ pour son traitement. Les médicaments prescrits ont été achetés à Santiago par le médecin à qui elle a remis la valeur à cet effet. Les services de radiologie ainsi que la mise en plâtre pour une fracture ont été payés, affirme une autre patiente dont l'enfant avait un bras cassé. Les analyses de laboratoire ont été effectuées à l'extérieur de l'hôpital. Pour les analyses et le plâtre, elle dit avoir dépensé 2820 pesos $(52,59 €)$.

\section{Discussion et conclusion}

Les résultats des entretiens montrent que le recours transfrontalier aux soins comme dans le cas de plusieurs autres pays (Bochaton, 2009, 2015 ; Dione, 2013 ; Durham, 2017 ; Liberona Concha et al., 2017) constitue une étape, parfois l'étape finale, d'un itinéraire thérapeutique débuté dans le pays d'origine des patientes ou patients. Il intervient souvent lorsque des résultats n'ont pas été obtenus dans le pays d'origine. Parfois, les patient.e.s décident $d u$ recours transfrontalier aux soins sans avoir transité au préalable par les structures de santé en Haïti. Dès lors traverser la frontière, indépendamment de son éloignement, apparaît comme "la solution » en particulier lorsque l'offre de l'autre côté semble plus attrayante. Pour ce faire, les patient.e.s doivent pouvoir mobiliser des compétences diverses comme la familiarité avec le système de santé, une pratique de la frontière, une connaissance de la langue ainsi que des ressources financières.

En ce sens, la situation en Haïti diffère peu de celles analysées dans d'autres contextes (Bochaton, 2009, 2015 ; Dione, 2013 ; Liberona Concha et al., 2017). Cependant, une particularité d'ordre ethnolinguistique est à souligner. En effet, contrairement aux autres situations frontalières, il existe une barrière linguistique. Celle-ci est contournée via un interprète qui sert de " passerelle entre les individus et les cultures »: il permet d'établir la communication, aux interlocuteurs de se comprendre et d'engager un dialogue (L. Tremblay, Brouillet, Rhéaume, \& Laquerre, 2006). L'interprète peut être soit un parent accompagnant le.la patient.e ou un employé de la clinique en République dominicaine. On est donc bien loin des facteurs signalés dans d'autres études, à savoir la langue comme un des éléments déterminants du retour par exemple des migrants mexicains dans leur pays d'origine pour la recherche de soins (Horton \& Cole, 2011 ; Lee et al., 2010). Malgré le recours à des interprètes, les barrières linguistiques et culturelles peuvent avoir des incidences sur les résultats des traitements, tels que signalés par un médecin dominicain (avec l'exemple d'une patiente ayant avalé un ovule). Or, ces barrières ne sont que peu évoquées dans la littérature sur la mobilité transfrontalière en santé. 

d'empathie, signalé par d'autres études réalisées en Haïti. En effet Tremblay (1995) décrit la distance dans les relations, lors des accouchements, le manque d'empathie, voire le mépris qui caractérise les relations entre patientes et médecins dans une zone non loin de Port-au-Prince, la capitale. Des années plus tard, dans une autre zone d'Haïti, les patient.e.s font part de leur vécu et soulignent le manque de considération, de prise en compte de leur problème. Parallèlement, ils et elles expriment une certaine satisfaction concernant l'accueil et les interactions avec les médecins dominicains des cliniques privées de Dajabón. Cependant, le travail de Minn (2004) offre un autre regard notamment en ce qui a trait au traitement réservé aux patient.e.s d'origine haïtienne à l'hôpital public de Dajabón. Selon cet auteur, la relation soigné.e.s/soignant.e.s à cet hôpital est méprisante. En ce sens, elle se rapproche de celle attribuée aux professionnels de santé des structures de soins en Haïti et dénoncée par les patient.e.s interviewées. Une étude plus approfondie sur cette question permettrait de comprendre comment les perceptions mutuelles Haïtiens/Dominicains influencent les interactions entre soignés/soignants.

Aucune femme ayant accouché à l'Hôpital Ramón Matías Mella de Dajabón n'a pu être interrogée, ce qui constitue une limite du travail. Il aurait été intéressant de pouvoir cerner les raisons qui conduisent les femmes haïtiennes à venir accoucher à Dajabón plutôt que de le faire à Ouanaminthe, mis à part le problème de disponibilité du service. Des femmes qui se dirigent vers des cliniques privées sont référées à l'hôpital à cause de leur difficulté économique. Ce choix de la part du médecin peut être perçu comme un acte de compassion (Minn, 2004), mais aussi comme une exigence éthique notamment dans des cas où une intervention est vitale.

Les réseaux sociaux, le bouche-à-oreille et la capacité à mobiliser des moyens financiers apparaissent comme des éléments importants non seulement dans la prise de décision et le choix du lieu de consultation. Les réseaux sociaux interviennent tant pour faciliter le passage de la frontière que dans la gestion de la thérapie. Les accompagnateurs et accompagnatrices servent aussi de support moral, de défenseur du patient en cas d'abus ou de mauvais traitement (Minn, 2004). Ainsi, le réseau social du patient ou de la patiente lui offre une certaine "garantie » en matière de sécurité, une "sécurité par le nombre » (Minn, 2004) pour traverser la frontière, mais aussi pour faire front au sein des structures hospitalières si nécessaire. Il s'agit d'une dimension peu explorée dans les études sur la mobilité transfrontalière en santé. Par ailleurs, l'effort financier consenti par les Haïtien.ne.s pour faire face aux dépenses de santé est important et peut être considéré comme un investissement si l'on considère le poids de la santé dans le capital humain. Or, une étude de la Banque mondiale et de l'Observatoire national de la pauvreté et de l'exclusion sociale (2014) souligne qu'en Haïti «les chocs idiosyncrasiques les plus importants sont liés à la santé » et qu'ils affectent notamment les populations plus vulnérables. Certains auteurs (Durham, 2017) signalent les différents mécanismes mis en place par les populations les plus pauvres pour faire face aux dépenses du recours transfrontalier aux soins. Transfert, vente d'actifs, aide de proches, identification de structures de soins publiques fournissant des soins gratuits, etc. sont parmi les stratégies mobilisées. Compte tenu, l'importance des transferts dans l'économie haïtienne, on peut penser qu'ils jouent un rôle dans la mobilité transfrontalière en santé. Cette dimension n'a cependant pas été explorée lors des entretiens et gagnerait à être creusée. La mobilité transfrontalière en santé, au regard 
de ces différents aspects, peut aussi contribuer au renforcement des inégalités dans l'accès à la santé.

Par ailleurs, une meilleure compréhension des comportements et des stratégies individuelles peut aider à la définition de politiques publiques en santé et à une allocation plus adéquate et équitable des ressources. De même, elle peut servir à l'élaboration d'un cadre de coopération bilatérale tenant compte des pratiques de mobilité transfrontalière en santé ainsi que l'épineuse question migratoire entre les deux pays.

\section{BIBLIOGRAPHIE}

Acosta, D. (2017, 25 agosto, 2017). República Dominicana registra más de 4 mil partos de mujeres haitianas. El Día. Retrieved from http://eldia.com.do

Allen, W. (2013). “I am From Busia!”: Everyday Trading and Health Service Provision at the Kenya-Uganda Border as Place-Making Activities. Journal of Borderlands Studies, 28(3), 291-306. doi:10.1080/08865655.2013.862756

Banque mondiale, \& Observatoire nationale de la pauvreté et de l'exclusion sociale. (2014). Haïti: investir dans l'humain pour combattre la pauvreté. Éléments de réflexion pour une prise de décision informée. Retrieved from Washington.

Benoist, J. (1993). Anthropologie médicale en société créole. Paris: Paris : Presses universitaires de France.

Bergmark, R., Barr, D., \& Garcia, R. (2010). Mexican Immigrants in the US Living Far from the Border may Return to Mexico for Health Services. Journal of Immigrant and Minority Health, 12(4), 610-614. doi:10.1007/s10903-008-9213-8

Bochaton, A. (2009). Construction d'un espace sanitaire transfrontalier: le recours aux soins des Laotiens en Thailande. (Doctorat Doctorat), Université de Nanterre - Parix X, Paris.

Bochaton, A. (2015). Cross-border mobility and social networks: Laotians seeking medical treatment along the Thai border. Social Science \& Medicine, 124, 364-373.

Bochaton, A., \& Lefebvre, B. (2008). The rebirth of the hospital: Heterotopia and medical tourism in Asia. In Asia on Tour: Exploring the rise of Asian tourism : Routledge.

Byrd, T. L., \& Law, J. G. (2009). Cross-border utilization of health care services by United States residents living near the Mexican border Utilización transfronteriza de los servicios de salud por residentes en los Estados Unidos que viven cerca de la frontera con México. Revista Panamericana de Salud Pública, 26(2), 95-100. doi:10.1590/S1020-49892009000800001

Caggiano, S. (2007). Madres en la frontera: género, nación y los peligros de la reproducción. Iconos. Revista de Ciencias Sociales(28).

Chrisman, N. J. (1977). The health seeking process: An approach to the natural history of illness. Culture, Medicine and Psychiatry, 1(4), 351-377. doi:10.1007/bfo0116243 
Connell, J. (2015). From medical tourism to transnational health care? An epilogue for the future. Social Science \& Medicine, 124, 398-401. doi:10.1016/j.socscimed.2014.11.015

Corten, A. (2011). L'État faible : Haïti et République dominicaine (Éd. rev. et augm.. ed.). Montréal: Montréal : Mémoire d'encrier.

De Jesus, M., \& Xiao, C. (2013). Cross-border health care utilization among the Hispanic population in the United States: implications for closing the health care access gap. Ethnicity \& Health, 18(3), 297-314. doi:10.1080/13557858.2012.730610

Diario Libre. (2017, 31 de Julio 2017). El director del Servicio Nacional de Salud dice que Haití y RD deben tratar la situación hospitalaria. Diario Libre. Retrieved from https://www.diariolibre.com Dilla Alfonso, H. (2004). Intercambio desigual y complejos urbanos binacionales en la frontera dominicana con Haití. Estudios fronterizos, 5, 35-58.

Dilla Alfonso, H. (2007). República Dominicana: La nueva cartografía transfronteriza. Caribbean Studies, 35(1), 181-205.

Dilla Alfonso, H. (2011). La migración transfronteriza haitiana en la República Dominicana. Retrieved from

Dilla Alfonso, H., Alexis, S., Antoine, M. I., Carmona, C., de Jesús Cedano, S., Murray, G. F., ... Sánchez, N. (2010). La frontera dominico-haitiana : Grupo de Estudios Multidisciplinarios Ciudades y Fronteras.

Dione, I. (2013). Polarisation des structures de soins de la Haute Casamance: entre construction nationale des systèmes de santé et recours aux soins transfrontalier. (Doctorat), Université d'Angers, Angers. (1320)

Droz, Y., Oro, A. P., \& Soares, E. (2014). Le butinage religieux: regard anthropologique sur les pratiques religieuses. In Mobilité religieuse. Retours croisés des Afriques aux Amériques. (pp. 17-37). Paris: Karthala.

Droz, Y., Soares, E., Gez, Y. N., \& Rey, J. (2016). La mobilité religieuse à l'aune du butinage. Social Compass, 63(2), 251-267. doi:10.1177/0037768616629305

Durham, J. (2017). Cross-border patient movement from the Lao PDR and the interplay between social networks and economic and cultural capital: A qualitative study. Asia Pacific Viewpoint, 58(2), 175-189. doi:10.1111/apv.12155

Fainzang, S. (1985). La « maison du blanc » : la place du dispensaire dans les stratégies thérapeutiques des Bisa du Burkina. Sciences Sociales et Santé, 105-128.

Fassin, D. (1990). Maladie et médecines.

Fassin, D., Jaffre, Y., Sociétés.

Fassin, D. (2001). La globalisation et la santé. Éléments pour une analyse anthropologique. In B. Hours (Ed.), Systèmes et politiques de santé. De la santé publique à l'anthropologie (pp. 24-40). Paris: Les Éditions Karthala.

Fosso, A. L. (2011). Typologie du pluralisme et butinage médical au Cameroun. Paper presented at the EASA Conference on medical pluralism, Rome, Italie.

Fouchard, J. (2017). Histoire d'Haïti. Tome I 1492 -1803 (Vol. Tome I). Port-au-Prince, Haïti: Éditions Henri Deschamps.

Glinos, I., Baeten, R., Helble, M., \& Maarse, H. (2010). A typology of cross-border patient mobility. Health \& Place, 16(6), 1145-1155. doi:10.1016/j.healthplace.2010.08.001 
Glinos, I., Doering, N., \& Maarse, H. (2012). Travelling home for treatment and EU patients' rights to care abroad: Results of a survey among German students at Maastricht University. Health Policy, 105(1), 38-45. doi:10.1016/j.healthpol.2011.12.008

González-Vázquez, T. T., Torres-Robles, C. A., \& Pelcastre-Villafuerte, B. E. (2013). Utilización transnacional de servicios de salud por parte de los migrantes mexicanos en Estados Unidos. Salud Pública de México, 55, s477-s484.

Guerrero, E., Donastorg, Y., \& de los Santos, A. (2014). Características y Análisi de salud de la población de origen extranjero -Estudio complementario de la primera Encuesta nacional de immigrantes de la República Dominicana ENI-2012. Retrieved from Santo Domingo, República Dominicana:

Hector, M., \& Hurbon, L. (2009a). Genèse de l'État haïtien (1804-1859). Paris : Éditions de la Maison des sciences de l'homme.

Hector, M., \& Hurbon, L. (2009b). Introduction. Les fondations. In M. Hector \& L. Hurbon (Eds.), Genèse de l'État haïtien (1804-1859). Paris: Éditions de la Maison des sciences de l'homme.

Herrera Javier, Lamaute-Brisson Nathalie, Milbin Daniel, Roubaud François, Saint-Macary Camille, Torelli Constance, \& Zanuso Claire. (2014). L'évolution des conditions de vie en Haïti entre 2007 et 2012. La réplique sociale du séisme. Paris, Port-au-Prince: IHSI, DIAL.

Horton, S., \& Cole, S. (2011). Medical returns: Seeking health care in Mexico. Social Science \& Medicine, 72(11), 1846-1852. doi:10.1016/j.socscimed. 2011.03.035

Institut Haïtien de l'Enfance -IHE/Haiti, \& ICF. (2018). Haiti Enquête Mortalité, Morbidité et Utilisation des Services 2016-2017 -EMMUS-VI. Retrieved from Pétion-Ville/Haïti:

Jan, J. M. (1950). Monographie religieuse des paroisses du Cap-Haïtien. Port-au-Prince, Haïti: H. Deschamps.

Janzen, J. M., \& Arkinstall, W. (1995). La quête de la thérapie au Bas-Zaire. Paris: Éditions Karthala.

Kangas, B. (2007). Hope from abroad in the international medical travel of Yemeni patients. Anthropology \& Medicine, 14(3), 293-305.

Laugesen, M. J., \& Vargas-Bustamante, A. (2010). A patient mobility framework that travels: European and United States- Mexican comparisons. Health Policy, 97(2- 3), 225-231. doi:10.1016/ j.healthpol.2010.05.006

Le National. (2017, 01 octobre 2017). Les ressortissants haïtiens interdits de se faire soigner dans des hôpitaux publics en République dominicaine. Le National. Retrieved from http:// www.lenational.org

Lee, J. Y., Kearns, R. A., \& Friesen, W. (2010). Seeking affective health care: Korean immigrants' use of homeland medical services. Health \& Place, 16(1), 108-115.

Liberona Concha, N., Tapia Ladino, M., \& Contreras Gatica, Y. (2017). Movilidad por salud entre Arica y Tacna: análisis de una demanda no satisfecha y de una oferta atractiva del otro lado de la frontera. GeopolíTica(S). Revista De Estudios Sobre Espacio Y Poder, 8(2), 253-278.

Luna, K. (2017, sábado, 26 de agosto de 2017). Haitianas ocupan mayoría de camas en cuidados intensivos Listin Diario. Retrieved from https://www.listindiario.com

Luna, K., Santana, R., Rodríguez, B., \& Durán, F. (2017, viernes 25 de agosto de 2017). Altos porcentajes -Caso parturientas de Haití preocupa a todas las regiones. Listin Diaro. Retrieved from https://www.listindiario.com 
Marcellini, A., Turpin, J.-P., Rolland, Y., \& Ruffié, S. (2000). Itinéraires thérapeutiques dans la société contemporaine. Le recours aux thérapies alternatives : une éducation à un « autre corps »? Corps et culture(5).

Ministère de la Santé Publique et de la Population. (2018). Rapport statistique 2017. Port-auPrince

Ley de presupuesto general del Estado 2017 - Tomo I, (2017a).

Ley de presupuesto general del Estado 2017 - Tomo I Informe explicativo política presupuestaria 2017, (2017b).

Minn, P. H. (2004). Health as a human right and medical humanitarianism on the HaitianDominican border. (Master of Arts), Mc Gill, Montreal.

Moya Pons, F. (2002). Manual de historia Dominicana (13. ed. ed.). Santo Domingo: Caribbean Publishers.

Moya Pons, F. (2009). La otra historia dominicana (Segunda edición ed.). Santo Domingo: Librería La Trinitaria.

Murray, G. F. (2010a). Dominican-Haitian Racial and Ethnic Perceptions and Sentiments: Mutual adaptations, mutual tensions, mutual anxieties. Retrieved from Dominican Republic:

Murray, G. F. (2010b). Sources of Conflict along and across the Haitian-Dominican border. Retrieved from Santo Domingo Dominican Republic:

Oficina Nacional de Estadistica. (2018). Segunda Encuesta Nacional de Inmigrantes en la República Dominicana -ENI-2017 -Version resumida del informe general. Santo Domingo: Oficina Nacional de Estadistica

Olivier de Sardan, J.-P. (2008). La rigueur du qualitatif : les contraintes empiriques de l'interprétation socio-anthropologique. Louvain-la-Neuve : Academia-Bruylant.

Ormond, M., \& Sulianti, D. (2017). More than medical tourism: lessons from Indonesia and Malaysia on South-South intra-regional medical travel. Current Issues in Tourism, 20(1), 94-110. doi:10.1080/13683500.2014.937324

Petrozziello, A. J., \& Wooding, B. (2014). Fanm Nan Fwontyè, Fanm Toupatou : Eclairage sur la violence exercée sur les Immigrantes d'origine haitienne, celles en transit migratoire et sur les déplacées internes le long de la frontière Dominicano-Haitienne (J. S. Roy, Trans.). Santo Domingo: Colectiva Mujer y Salud, Mujeres del Mundo et Observatoire sur la migration et la Caraibe (Observatory Migrants of the Caribbean) (CIES-UNIBE).

Poschet El Moudden, L. (2006). Villes à la frontière et transformation de l'espace : Le cas de Haïti et la République Dominicaine. (Docteur ès Sciences), École Polytechnique Fédérale de Lausanne, Lausanne. (3655)

Price-Mars, J. (1953). La République d'Haïti et la République dominicaine. Les aspects divers d'un problème d'histoire, de géographie et d'ethnologie. TOME I (Vol. Tome I). Port-au-Prince : Collection du Tricinquantenaire de l'Indépendance d'Haïti.

Rathe, M., \& Moliné, A. (2011). Sistema de salud de República Dominicana. Salud Pública de México, 53, s255-s264.

Rivadulla, M. (2017, 1 de septiembre de 2017). Parturientas haitianas. Acento.

Saint-Pré, P. (2017, 2017-09-21 ). Les Dominicains menacent de ne plus soigner les Haïtiens gratuitement Le Nouvelliste. Retrieved from https://lenouvelliste.com 
Saint-Pré, P. (2018, 2018-01-09). 80\% des étrangères qui enfantent chaque année en République dominicaine sont haïtiennes Le Nouvelliste. Retrieved from https://lenouvelliste.com

Sakoyan, J. (2012). Les mobilités thérapeutiques -Bilan et perspectives depuis les Comores. Anthropologie et Santé(5).

Sandelowski, M., \& Barroso, J. (2006). Handbook for synthesizing qualitative research : Springer Publishing Company.

Santiago Santana, R. (2017). 40 emigrantes en total : Interceptan a 15 embarazadas venían de Haití Listin Diario. Retrieved from https://listindiario.com

Servicio Nacional de Salud. (2017). Memoria Institucional. República Dominicana: Servicio Nacional de Salud (SNS)

Soares, E. (2009). Le butinage religieux: pratiques et pratiquants au Brésil : KARTHALA Editions. Soares, E. (2011). Religious “Butinage”. Looking at Paranaguá-mirim (Brazil). Social Compass, 58(2), 223-234. doi:10.1177/0037768611402620

Théodat, J.-M. (2003). Haïti-République Dominicaine : une île pour deux, 1804-1916 : Paris : Éditions Karthala.

Théodat, J.-M., Mathon, D., Mathelier, R., \& Casséus, M. (2003). Quisqueya: un papillon d'envol. In R. Mathelier, D. Mathon, \& M. Casséus (Eds.), Entreprise, Territoire et Développement: Compilation 2002-2003. Port-au-Prince: INESA/Le Nouvelliste.

Tremblay, J. (1995). Mères, pouvoir et santé en Haiti. Paris: Paris Karthala.

Tremblay, L., Brouillet, M.-I., Rhéaume, J., \& Laquerre, M.-E. (2006). La communication avec interprète dans l'intervention à domicile : Centre de recherche et de formation, CSSS de la Montagne.

Wismar, M., Palm, W., Figueras, J., Ernst, K., \& Van Ginneken, E. (2011). Cross-border health care in the European Union: mapping and analysing practices and policies. Cross-border health care in the European Union: mapping and analysing practices and policies.

Wooding, B. (2012). Women fight for their safety in the Dominican-Haitian border. Migration and Development, 10(18), 37-58.

World Health Organization. (2018, 2018-02-07). Health Workers density and distribution. Skilled health professionals. Retrieved from http://apps.who.int/gho/data/node.sdg.3-c-viz?lang=en

\section{NOTES}

1. En 2017 le taux moyen était : 1 euro $=53,62$ pesos ; 1 dollar américain $=47,54$ pesos.

2. Ces tensions découlent autant de mesures phytosanitaires prises par les autorités haïtiennes lesquelles visaient à prohiber l'importation d'œufs et de produits carnés en provenance de la République dominicaine que de la résurgence d'une nouvelle vague anti-haïtienne. Le verdict TC/168 de septembre 2013 de la Cour constitutionnelle contre plus de 200 mille descendants d'immigrants haïtiens tend aussi par ailleurs à exacerber les manifestations de xénophobie, à accroître les tensions, les contrôles frontaliers ainsi que les rapatriements. 


\section{RÉSUMÉS}

Cette étude aborde la mobilité thérapeutique transfrontalière entre deux pays à faibles revenus, Haïti et la République dominicaine. L'objectif est d'explorer les logiques qui sous-tendent la recherche de soins des Haïtiens et Haïtiennes résidant dans la ville frontalière de Ouanaminthe. En plus de cerner les motivations du recours transfrontalier aux soins de santé, l'étude qualitative propose de le situer dans l'itinéraire thérapeutique. Vingt et un entretiens semidirigés auprès de patientes et patients ont été menés dans deux cliniques privées et un hôpital public à Dajabón (République dominicaine). Sept entretiens ont aussi été menés avec des cliniciens et gestionnaires. Ces récits révèlent que la recherche d'efficacité et de qualité, la relation patient/médecin et le temps d'attente interviennent dans la décision de se faire soigner en République dominicaine. Le cheminement thérapeutique des patientes et patients n'est pas linéaire, mais fait de va-et-vient, selon les besoins, entre deux systèmes de santé originellement disjoints.

\section{Background}

On 16 September 2017, 40 Haitian, including 15 pregnant Haitian women, were arrested at the Dominican border and repatriated. This information relayed by the Haitian press echoes those published by some Dominican newspapers, which repeatedly denounce the care provided by Dominican public hospitals to Haitian patients and its impact on the health care system. Nevertheless, very few studies in Haiti or the Dominican Republic have addressed this issue even though several authors have pointed out the existence of health care related cross-border mobility. Moreover, the reasons for such mobility are rarely analyzed. For some, the lack of services in Haiti and free access to health care in the Dominican Republic are identified as the main drivers for such behavior. Yet crossing the border (even at official entry points) is not always easy for Haitians. Our paper asks: what then are the driving factors that lead to crossborder health care seeking? At what point in the therapeutic itinerary is such a decision made?

Evidence from studies on transnational health related mobility indicates that availability, affordability, accessibility, familiarity and perception of service quality play a role in the decision-making. Furthermore, in a context of proximity with the border, attractiveness of care can polarize the flows in a push/pull dynamics. Several studies also highlight the importance of social networks and ethnolinguistic factors in the decision to travel or return to their country of origin after care has been sought. Dissatisfaction with local services and hope for better care are also among the identified driving factors. This lead to complex and sinuous therapeutic paths within and outside one's country.

The main purpose of this paper is to explore the rationale behind the health-related cross-border mobility of Haitians living in or near the border town of Ouanaminthe, Haiti. The driving factors will also be identified and cross-border health utilization will be contextualized within the overall therapeutic itinerary.

\section{Methods}

This descriptive qualitative study used several combined methods, namely observation and semidirective interviews. Overall, 28 interviews were carried out (21 patients, 7 Dominican and Haitian clinicians or decision makers). The interviews with cross-border patients focused mainly on their therapeutic itinerary, the motives behind this decision, obstacles encountered and their perceptions of the quality of care received both in Haiti and the Dominican Republic. Interviews with the clinicians and decision makers helped gathered information on health care facilities, their attendance by Haitian clients and the existing collaboration or system of medical referral 
between Haiti and the Dominican Republic.

An iterative thematic analysis process was conducted. First, a pre-coding based and initial thematic categories inspired from the literature was used. From this process, other thematic categories have emerged, pointing to context specific issues.

\section{Findings}

Patients' cross-border health seeking is not linear, but rather a back and forth process between two separate health care systems. For some participants, it lasts several years, while for others, it leads to a first visit. The results show that visiting different health care facilities for the same health problem (or evolving symptoms over time) is usual. Having a second opinion is a frequently reported reason (for both patients and clinicians). The study also shows that crossborder health seeking requires participants to mobilize various skills such as familiarity with the health care system, border crossing experience and abilities, and knowledge of the Dominican language. Respondents also emphasized three main issues related to the Haitian health system that led them to cross-border for health care in the Dominican Republic: 1) the search for efficiency and service quality; 2) the poor patient/clinician relationship; 3) excessive waiting times. Furthermore, the importance of social networks and the strategies used to overcome language barrier is striking. Both clinicians and patients implement different strategies to enable appropriate care. Participants also underlined the important financial burden required to seek treatment abroad.

\section{Conclusion}

The cross-border health-related mobility practices observed in Ouanaminthe have many similarities with those analyzed in other contexts. Some aspects have been highlighted but need to be deepened to gain a better understanding of the health-seeking behavior as well as the strategies in place to cope with the expenses. An in-depth understanding of individual behaviors and strategies can help in the definition of public health policies and the development of a framework for a bilateral cooperation that takes into account cross-border health mobility practices and the thorny issue of migration.

\section{INDEX}

Mots-clés : mobilité thérapeutique, accès aux soins, frontière

Keywords : therapeuthic mobility, health care access, boundary

Index géographique : Haïti, République dominicaine

\section{AUTEURS}

\section{DOMINIQUE MATHON}

Laboratoire d'équité environnementale, INRS Centre Urbanisation Culture Société, Canada

PHILIPPE APPARICIO

Laboratoire d'équité environnementale, INRS Centre Urbanisation Culture Société, Canada

\section{UGO LACHAPELLE}

Université du Québec à Montréal (UQAM), Canada 